\title{
UPDATED OKLAHOMA OZARK FLORA A Checklist for the Vascular Flora of Ozark Plateau in Oklahoma based on the work of C.S. Wallis and records from the Oklahoma Vascular Plants Database
}

\author{
Bruce W. Hoagland \\ Oklahoma Biological Survey and Department of Geography \\ University of Oklahoma, Norman, OK 73019-0575 \\ e-mail: bhoagland@ou.edu
}

Charles Wallis' 1959 dissertation

"Vascular Plants of the Oklahoma

Ozarks" is one of the most important florisitic works for state botanists and conservationists. Although a number of local and county floras for Oklahoma have been published, only Wallis and C. T. Eskew (1937) have completed regional studies. Wallis's interest in the Ozark flora began with his 1953 masters thesis, "The Spermophyta of Cherokee County Oklahoma," and subsequent studies in collaboration with U. T. Waterfall at Oklahoma A\&M (Wallis 1957; Wallis and Waterfall 1953; Waterfall and Wallis 1962, 1963). This paper has two objectives, to update the taxonomy of Wallis's Ozark list (WOL) and to provide a current Ozark checklist (OC) by inclusion of records that did not appear in WOL. Since several decades have passed since the WOL was completed, there have been many changes in the taxonomy of the plants listed. These updates will enhance the utility of the WOL for modern users and not detract from Wallis's original work.

The OC was compiled by comparing the updated WOL with the Oklahoma Vascular Plants Database (OVPD; Hoagland et al. 2007). Nomenclature for the OC follows the United States Department of Agriculture-Natural Resources Conservation Service (USDANRCS 2007). In the OC, species introduced to North America were determined using the USDA-NRCS (2007).

The WOL and OC were summarized separately following Palmer et al. (1995) (Tables 1 and 2). The OC was also compared with the rare species tracking list of the Oklahoma Natural Heritage Inventory (2007) to determine which species of conservation interest were listed (Table 3).

The WOL consisted of 1,205 species or 1,240 when subspecies, varieties, and hybrids were added. These taxa belong to 556 genera in 131 families. In the OC, there were 303 species that did not appear in the WOL, for a total of 1,508 species. Subspecies, varieties, and hybrids accounted for 57 additional taxa, increasing the total to 1,565 taxa. (Note that the OC does not include Castanea dentata, Opuntia phaeacantha, and Quercus coccinea species which appeared in Wallis's original list. They have since been annotated to other taxa.) These taxa belong to 615 genera in 145 families. The most speciose families in the WOL were the Asteraceae 213, Poaceae 172, Cyperaceae 104, and Fabaceae 100. The genus Carex contained the most species (51) in the WOL, followed by Dichanthelium and Polyogonum, each with 20 taxa.

There were 134 taxa of non-native plants or $10.8 \%$ of the total taxa in the WOL. There were an additional 54 non-native taxa in the OC for a total of 188 , or $12.0 \%$ of the total taxa reported. Non-native species occurred in 45 families. The genera with the greatest number of non-native species were Trifolium (7 species), Bromus (5), and Polygonum (5). 
Oklahoma Native Plant Record Volume 7, Number 1, December 2007

Seventy-nine taxa tracked by the Oklahoma Natural Heritage Inventory were present in the OC (Table 3). Of these, 50 were reported by Wallis and 28 were added from the OVPD.

Conservation ranks are assigned to taxa according to level of imperilment at the state $(S)$ and global $(G)$ levels on a scale of $1-5$, where 1 represents a species that is imperiled and 5 a species that it is secure (Groves et al. 1995). Fifty-one taxa or $66.4 \%$ of those in Table 3 were ranked as G5 and thus considered demonstrably secure at the global scale. No taxa were ranked G1 or G2, indicating imperilment at the global level. Thirty-one taxa (39.7\%), however, were ranked as S1, 12 as S2, and 19 as S1S2. The higher percentage of state rare species indicates that many of these species are at the western margin of their ranges in eastern Oklahoma. In Oklahoma, some of species listed in Table 3 occur only in the Ozarks, such as Clematis virginiana, Equisetum arvense (one location in Adair County), Erigenia bulbosa, Gentiana alba, Glyceria acutiflora, Heteranthera dubia (one location in Cherokee County), Physocarpus opulifolius var. intermedius, Silene regia, and Symphyotrichum novae-angliae (Cherokee County only). Castanea pumila var. ozarkensis and Silene regia are species of concern and were once candidates for federal listing as threatened.

\section{Literature Cited}

Eskew, C.T. 1937. The flowering plants of the Wichita National Forest. M.S. thesis, University of Oklahoma, Norman.

Groves, C.R., M.L. Klein, and T.F. Breden. 1995. Natural heritage programs: public-private partnerships for biodiversity conservation. Wildlife Society Bulletin 23: 784-790.

Hoagland, B.W., A.K. Buthod, I.H. Butler, P. Callahan-Crawford, W.E.
Elisens, and R. Tyrl. 2007. Oklahoma Vascular Plants Database. University of Oklahoma, Norman. www.biosurvey.ou.edu accessed 1 October 2007).

Oklahoma Natural Heritage Inventory. 2007. Oklahoma Natural Heritage Inventory working list of rare Oklahoma plants. University of Oklahoma, Norman. (www.biosurvey.ou.edu/publicat.html accessed 1 October 2007).

Palmer, M.W., G.L. Wade, and P. Neal. 1995. Standards for the writing of floras. Bioscience 45: 339-345.

USDA-NRCS. 2007. The PLANTS Database National Plant Data Center, Baton Rouge, LA 70874-4490 USA(http://plants.usda.gov accessed 1 May 2007).

Wallis, C.S. 1953. The Spermatophyta of Cherokee County, Oklahoma (exclusive of the Poaceae, Cyperaceae, and Juncaceae). M.S. thesis, Oklahoma A\&M College, Stillwater, Oklahoma.

Wallis, C.S. 1957. Additions to the Oklahoma flora from the Oklahoma Ozarks. Proc. Oklahoma Acad. Sci. 38: 3-5.

Wallis, C.S. 1957. Additions to the Oklahoma flora from the Oklahoma Ozarks. Proc. Oklahoma Acad. Sci 38: 3-5.

Wallis, C.S. 1959. Vascular plants of the Oklahoma Ozarks. Ph.D dissertation, Oklahoma State University, Stillwater, Oklahoma.

Wallis, C.S. and U.T. Waterfall. 1953. Additions to the Oklahoma flora from Cherokee County. Proc. Oklahoma Acad. Sci. 34: 124-125.

Waterfall, U.T. and C.S. Wallis. 1962. Some geographic relationships of the vascular flora of the Oklahoma Ozarks: Studies in the composition and distribution of the Oklahoma flora. Proc. Oklahoma Acad. Sci. 43: 61-63.

Waterfall, U.T. and C.S. Wallis. 1963. A list of the vascular flora of the Oklahoma Ozarks. Proc. Oklahoma Acad. Sci. 44: 11-22. 
Oklahoma Native Plant Record

Volume 7, Number 1, December 2007

Table 1 Summary of Wallis's (1959) floristic list of the Oklahoma Ozarks. Numbers outside the parentheses represent the number of species reported, those within the parentheses represent the total number of taxa reported, including subspecies and varieties. The number of hybrids reported is denoted with an asterisk.

\begin{tabular}{lccc}
\hline Taxonomic Group & Taxa & Native & $\begin{array}{c}\text { Non- } \\
\text { native }\end{array}$ \\
\hline Equisetophyta & $0\left(1^{*}\right)$ & $0(1 *)$ & 0 \\
Lycopodiophyta & 1 & 1 & 0 \\
Pteridophyta & 21 & 21 & 0 \\
Coniferophyta & 2 & 2 & 0 \\
Magnoliophyta & 1,181 & 1,047 & 134 \\
& & $(1,081)$ & \\
Magnoliopsida & $882(909)$ & $(808)$ & 101 \\
& & 266 & \\
Liliopsida & $299(306)$ & $(273)$ & 33 \\
\hline Total & 1,205 & 1,071 & \\
& $(1,240)$ & $(1,106)$ & 134 \\
\hline
\end{tabular}

Table 2 Summary of all plants reported from the Oklahoma Ozarks based upon Wallis (1959) and data in the Oklahoma Vascular Plants Database. Numbers outside the parentheses represent the number of species reported, those within the parentheses represent the total number of taxa reported, including subspecies and varieties. The number of hybrids reported is denoted with an asterisk.

\begin{tabular}{|c|c|c|c|}
\hline $\begin{array}{l}\text { Taxonomic } \\
\text { Group }\end{array}$ & Taxa & Native & $\begin{array}{l}\text { Non- } \\
\text { native }\end{array}$ \\
\hline Equisetophyta & $3(3 ; 1 *)$ & $3(3 ; 1 *)$ & 0 \\
\hline Lycopodiophyta & 3 & 3 & 0 \\
\hline Pteridophyta & $31(32)$ & $31(32)$ & 0 \\
\hline Coniferophyta & 3 & 3 & 0 \\
\hline Magnoliophyta & $\begin{array}{c}1,481 \\
(1,518 \\
\left.5^{*}\right)\end{array}$ & $\begin{array}{c}1,280 \\
(1,330 \\
\left.5^{*}\right)\end{array}$ & 188 \\
\hline Magnoliopsida & $\begin{array}{c}1,082 \\
(1,125 ; \\
\left.5^{*}\right)\end{array}$ & $\begin{array}{c}945(983 ; \\
\left.5^{*}\right)\end{array}$ & 142 \\
\hline Liliopsida & $381(393)$ & $335(347)$ & 46 \\
\hline Total & $\begin{array}{c}1,508 \\
(1,565)\end{array}$ & $\begin{array}{c}1,321 \\
(1,377)\end{array}$ & 188 \\
\hline
\end{tabular}

Table 3 Species tracked by the Oklahoma Natural Heritage Inventory in the Oklahoma Ozarks. This list is a combination of Wallis (1959) and records from the Oklahoma Vascular Plants Database. Taxa not reported in Wallis 1959 are denoted with \#. Taxa are ranked according to level of imperilment at the state (S) and global $(G)$ levels on a scale of $1-5$, where 1 represents a species that is imperiled and 5 a species that it is secure (Groves et al. 1995).

\begin{tabular}{|c|c|c|}
\hline Taxa & $\begin{array}{c}\text { G- } \\
\text { rank }\end{array}$ & $\begin{array}{c}\text { S- } \\
\text { rank }\end{array}$ \\
\hline Agalinis tenuifolia var. parviflora & G5 & $\mathrm{S} 2 \mathrm{~S} 3$ \\
\hline \#Agalinis viridis & G4 & $\mathrm{S} 1$ \\
\hline Aletris farinose & G5 & S1S2 \\
\hline Arabis shortii & $\begin{array}{l}\text { G5 } \\
\text { G4 }\end{array}$ & S1S2 \\
\hline Arnoglossum atriplicifolium & G5 & S1S2 \\
\hline Arnoglossum reniforme & G4 & S1S3 \\
\hline Aruncus dioicus var. pubescens & G5 & S1S3 \\
\hline Asplenium bradleyi & G4 & S1 \\
\hline Axonopus fissifolius & G5 & S1 \\
\hline \#Brachyelytrum erectum & G5 & S1 \\
\hline \#Brasenia schreberi & G5 & S1 \\
\hline Callirhoe bushii & G3 & S3 \\
\hline \#Calopogon oklahomensis & G4 & S1 \\
\hline Calopogon tuberosus var. tuberosus & G5 & S1 \\
\hline Carex cephalophora var. cephalophora & G5 & S2 \\
\hline Carex oklahomensis & G4 & S2 \\
\hline \#Carex oxylepis & G5 & S2 \\
\hline Castanea pumila var. ozarkensis & G5 & S2 \\
\hline \#Cayaponia grandifolia & G4 & S1 \\
\hline Cladrastis kentukea & G4 & $\mathrm{S} 2 \mathrm{~S} 3$ \\
\hline Clematis virginiana & G5 & S1S2 \\
\hline \#Collinsia verna & G5 & S1 \\
\hline \#Corallorrbiza odontorbiza & G5 & S1 \\
\hline
\end{tabular}




\begin{tabular}{|c|c|c|c|c|c|}
\hline Cotinus obovatus & G4 & S3 & Panicum bracbyanthum & G5 & $\mathrm{S} 2 \mathrm{~S} 3$ \\
\hline \#Croton michauxii & G5 & S1 & \#Perideridia americana & G4 & S1S2 \\
\hline \#Cypripedium kentuckiense & G3 & S1 & \#Phacelia gilioides & G5 & S1 \\
\hline Desmodium pauciflorum & G5 & S1 & Phaseolus polystachios & G4 & S1 \\
\hline Dicentra cucullaria & G5 & S1S2 & Philadelphus pubescens & G5 & S2 \\
\hline Draba aprica & G3 & S1 & Physocarpus opulifolius var.intermedius & G5 & S1S3 \\
\hline \#Drosera brevifolia & G5 & S2S3 & \#Pilularia americana & G5 & S1S2 \\
\hline \#Equisetum arvense & G5 & S1 & Platanthera lacera & G5 & S1S2 \\
\hline \#Erigenia bulbosa & G5 & S1S2 & \#Podostemum ceratophyllum & G5 & S2 \\
\hline Fraxinus quadrangulata & G5 & S2S3 & Rhamnus lanceolata ssp. glabrata & $\begin{array}{l}\text { G5 } \\
\text { G4 }\end{array}$ & S1 \\
\hline Galium arkansanum & G5 & S1S2 & \#Rhus lanceolata & G5 & S1S2 \\
\hline \#Gentiana alba & $\begin{array}{l}\text { G4 } \\
\text { G4 }\end{array}$ & S1 & Rhododendron canescens & G5 & S2S3 \\
\hline \#Gentiana puberulenta & G5 & S1 & \#Ribes missouriense & G5 & S1 \\
\hline Glyceria acutiflora & G5 & S1 & Rorippa teres & G5 & S1S2 \\
\hline Hedeoma pulegioides & G5 & S1S3 & Silene regia & G3 & S1 \\
\hline Heteranthera dubia & G5 & S2 & Sporobolus vaginiflorus var. ozarkanus & G5 & S1S2 \\
\hline Hexalectris spicata & G5 & S1S2 & Symphyotrichum laeve var. laeve & G5 & S1S3 \\
\hline Hypericum gentianoides & G5 & S1S2 & Symphyotrichum novae-angliae & G5 & S1 \\
\hline Impatiens pallida & G5 & S2 & Tilia americana var. americana & G5 & S1S2 \\
\hline Iris cristata & G5 & S2 & Tilia americana var. caroliniana & $\begin{array}{l}\text { G5 } \\
\text { G4 }\end{array}$ & S1S2 \\
\hline Iris virginica & G5 & S2 & \#Tipularia discolor & $\begin{array}{l}\text { G5 } \\
\text { G3 }\end{array}$ & S1 \\
\hline \#Malaxis unifolia & G5 & S1 & Tradescantia ernestiana & G4 & S? \\
\hline \#Marsilea vestita & G5 & S1 & Tradescantia ozarkana & G3 & S1S2 \\
\hline \#Monotropa hypopithys & G5 & S1 & Ulmus serotina & G4 & S2 \\
\hline \#Monotropa uniflora & G5 & S1 & Urtica dioica & G5 & S2 \\
\hline \#Mublenbergia bushii & G5 & S1S2 & Uvularia grandiflora & G5 & S2S3 \\
\hline Neobeckia aquatica & $\begin{array}{l}\text { G4 } \\
\text { G3 }\end{array}$ & S1S3 & Valerianella ozarkana & G3 & S1 \\
\hline \#Panax quinquefolius & G4 & S1 & & & \\
\hline
\end{tabular}

Hoagland, B.W. 
Appendix: a checklist for the vascular flora of Ozark Plateau in Oklahoma. This list was compiled from Wallis (1959) with additions from the Oklahoma Vascular Plant Database (Hoagland et al. 2007). \# Indicates species not appearing in Wallis (1959). * Indicates species that are not native to North America are marked with an asterisk.

\section{EQUISETOPHYTA}

Equisetaceae

\#Equisetum arvense L.

Equisetum $\times$ ferrissii Clute (pro sp.) [hyemale $\times$ laevigatum]. Syn. $=$ Equisetum hymenale

L. var. intermedium.

\#Equisetum hyemale L.

\#Equisetum laevigatum A. Braun

\section{LYCOPODIOPHYTA} Isoetaceae

\#lsoetes melanopoda Gay \& Durieu ex Durieu

\section{Selaginellaceae}

Selaginella apoda (L.) Fern.

\#Selaginella rupestris (L.) Spring

\section{PTERIDOPHYTA}

Aspleniaceae

Asplenium bradleyi D.C. Eat.

Asplenium platyneuron (L.) B.S.P.

Asplenium resiliens Kunze

Asplenium rhizophyllum L. Syn. =

Camptosorus rhizophyllus (L.) Link.

\section{Dryopteridaceae}

Athyrium filix-femina (L.) Roth ssp.

asplenioides (Michx.) Hultén. Syn. = A.

filix-femina (L.) Roth var. asplenioides

(Michx.) Farw.

\#Cystopteris bulbifera (L.) Bernh.

Cystopteris fragilis (L.) Bernh. var. fragilis.

Wallis listed forma dentata (Dickson) Clute

Cystopteris tennesseensis Shaver. Syn. $=C$.

fragilis (L.) Bernh. var. simulans

(Weatherby) McGregor.

Dryopteris marginalis (L.) Gray

Onoclea sensibilis $\mathrm{L}$.

Polystichum acrostichoides (Michx.) Schott

Woodsia obtusa (Spreng.) Torr.
Marsileaceae

\#Marsilea vestita Hook. \& Grev.

\#Pilularia americana A. Braun

Ophioglossaceae

\#Botrychium dissectum Spreng.

Botrychium virginianum (L.) Sw.

\#Ophioglossum crotalophoroides Walt.

\#Ophioglossum engelmannii Prantl

\section{Polypodiaceae}

\#Pleopeltis polypodioides (L.) Andrews \& Windham ssp. michauxiana (Weatherby) Andrews \& Windham

\section{Pteridaceae}

Adiantum capillus-veneris L.

Adiantum pedatum $\mathrm{L}$.

Argyrochosma dealbata (Pursh) Windham.

Syn. $=$ Notholaena dealbata (Pursh)

Kunze.

Asplenium trichomanes $\mathrm{L}$.

Cheilanthes alabamensis (Buckl.) Kunze

Cheilanthes lanosa (Michx.) D.C. Eat. Syn. =

C. vestita (Spreng.) Sw.

\#Cheilanthes tomentosa Link

Pellaea atropurpurea (L.) Link

\#Pellaea wrightiana Hook.

\#Pteridium aquilinum (L.) Kuhn var. latiusculum

(Desv.) Underwood ex Heller

Pteridium aquilinum (L.) Kuhn var. pseudocaudatum (Clute) Heller

\section{Thelypteridaceae}

Phegopteris hexagonoptera (Michx.) Fée. Syn. = Dryopteris hexagonoptera (Michx.) C. Christens.

\#Thelypteris palustris Schott var. pubescens (Lawson) Fern. 
CONIFEROPHYTA

Cupressaceae

\#Juniperus ashei Buchh. Juniperus virginiana $\mathrm{L}$.

Pinus echinata P. Mill.

\section{Pinaceae}

\section{MAGNOLIOPHYTA} MAGNOLIOPSIDA

Acanthaceae

Dicliptera brachiata (Pursh) Spreng.

Justicia americana (L.) Vahl.

\#Ruellia caroliniensis (J.F. Gmel.) Steud. ssp. ciliosa (Pursh) R.W. Long var. cinerascens (Fern.) Kartesz \& Gandhi

Ruellia humilis Nutt. Syns. $=R$. humilis Nutt. var. expansa Fern. and $R$. humilis Nutt. var. longiflora (Gray) Fern.

Ruellia pedunculata Torr. ex Gray

Ruellia strepens L.

\section{Aceraceae}

Acer negundo L. var. negundo Acer negundo L. var. texanum Pax Acer rubrum $\mathrm{L}$. Acer saccharinum $\mathrm{L}$. Acer saccharum Marsh.

\section{Amaranthaceae}

Amaranthus albus L. \#Amaranthus arenicola I.M. Johnston Amaranthus graecizans $\mathrm{L}$. Amaranthus hybridus L. \#Amaranthus palmeri S. Wats. Amaranthus retroflexus $L$. Amaranthus spinosus $\mathrm{L}$. Amaranthus tuberculatus (Moq.) Sauer. Syn. = Acnida tamariscina auct. non (Nutt.) Wood

Froelichia floridana (Nutt.) Moq. var. campestris (Small) Fern.

Froelichia graçilis (Nutt.) Moq. Iresine rhizomatosa Standl.

Cotinus obovatus Raf.
Rhus aromatica Alt. var. aromatica

Rhus aromatica Alt. var. serotina (Greene)

Rehd.

Rhus copallinum L. var. latifolia Engl.

Rhus glabra L.

\#Rhus lanceolata (Gray) Britt.

\#Rhus trilobata Nutt.

\#Rhus trilobata Nutt. var. simplicifolia (Greene) Barkl.

Toxicodendron rydbergii (Small ex Rydb.) Greene. Syn. = Rhus radicans $\mathrm{L}$. var. vulgaris (Michx.) DC. Wallis listed formas negundo (Greene) Fern. and vulgaris.

Toxicodendron pubescens $\mathrm{P}$. Mill. Syn. = Rhus toxicodendron $\mathrm{L}$.

\section{Anonaceae}

Asimina triloba (L.) Dunal

Apiaceae (= Umbelliferae)

\#Ammoselinum butleri (Engelm. ex S. Wats.) Coult. \& Rose

*\#Anethum graveolens $\mathrm{L}$.

Angelica venenosa (Greenway) Fern.

\#Bifora americana Benth. \& Hook. f. ex S. Wats.

Chaerophyllum procumbens (L.) Crantz

\#Chaerophyllum tainturieri Hook. var. dasycarpum Hook. ex S. Wats.

Chaerophyllum tainturieri Hook. var. tainturieri.

Syn. = C. texanum Coult. \& Rose.

Cicuta maculata L.

Cryptotaenia canadensis (L.) DC.

*Daucus carota L. Wallis listed formas carota and epurpuratus Farw.

Daucus pusillus Michx.

\#Erigenia bulbosa (Michx.) Nutt. Eryngium leavenworthii Torr. \& Gray \#Eryngium prostratum Nutt. ex DC.

Eryngium yuccifolium Michx. var. synchaetum

Gray ex Coult. \& Rose

Hydrocotyle verticillata Thunb.

Limnosciadium pinnatum (DC.) Mathias \&

Constance

Osmorhiza longistylis (Torr.) DC. Syn. =

Hoagland, B.W. 
Osmorhiza longistylis (Torr.) DC. var. villicaulis Fern.

Oxypolis rigidior (L.) Raf.

\#Perideridia americana (Nutt. ex DC.)

Reichenb.

Polytaenia nuttallii DC.

\#Ptilimnium capillaceum (Michx.) Raf.

\#Ptilimnium nuttallii (DC.) Britt.

Sanicula canadensis $L$. var. canadensis

Sanicula odorata (Raf.) K.M. Pryer \& L.R.

Phillippe. Syn. = S. gregaria Bickn.

Spermolepis divaricata (Walt.) Raf. ex Ser.

Spermolepis echinata (Nutt. ex DC.) Heller

Taenidia integerrima (L.) Drude

Thaspium barbinode (Michx.) Nutt.

Thaspium trifoliatum (L.) Gray var. aureum

Britt. Syn. $=T$. trifoliatm (L.) Gray var.

flavum Blake.

*\#Torilis arvensis (Huds.) Link

${ }^{*}$ Torilis japonica (Houtt.) DC.

Trepocarpus aethusae Nutt. ex DC.

Zizia aptera (Gray) Fern.

Zizia aurea (L.) W.D.J. Koch

Amsonia illustris Woods.

Amsonia tabernaemontana Walt. var. salicifolia

(Pursh) Woods.

Amsonia tabernaemontana Walt. var.

tabernaemontana

Apocynum androsaemifolium $\mathrm{L}$.

Apocynum cannabinum L. Syn. $=A$.

cannabinum L. var. glaberrimum A. DC.

and Apocynum cannabinum L. var.

pubescens (Mitchell ex R. Br.) Woods.

Ilex decidua Walt.

\section{Aquifoliaceae}

Araliaceae
\#Panax quinquefolius L.

Aristolochiaceae

\#Aristolochia serpentaria L.

Aristolochia tomentosa Sims

Asarum canadense $\mathrm{L}$. Syn. $=$ A. canadense $\mathrm{L}$. var. acuminatum Ashe.

\author{
Asclepiadaceae \\ Asclepias amplexicaulis Sm. \\ Asclepias hirtella (Pennell) Woods. \\ Asclepias incarnata L. ssp. incarnata \\ Asclepias obovata Ell. \\ Asclepias purpurascens $\mathrm{L}$. \\ Asclepias quadrifolia Jacq. \\ Asclepias stenophylla Gray \\ Asclepias sullivantii Engelm. ex Gray \\ Asclepias tuberosa L. ssp. interior Woods. \\ \#Asclepias variegata $\mathrm{L}$ \\ Asclepias verticillata $\mathrm{L}$. \\ Asclepias viridiflora Raf. Syn. $=$ A . viridiflora \\ Raf. var. lanceolata (Ives) Torr. and $A$. \\ viridiflora Raf. var. linearis (Gray) Fern. \\ Asclepias viridis Walt. \\ Cynanchum laeve (Michx.) Pers. \\ Matelea baldwyniana (Sweet) Woods. \\ Matelea decipiens (Alexander) Woods. \\ Matelea gonocarpos (Walt.) Shinners
}

Asteraceae (= Compositae)
Achillea millefolium L. var. occidentalis DC.

Syn. = A. lanulosa Nutt. Wallis listed formas lanulosa and rubicunda Farwell.

Ageratina altissima (L.) King \& H.E. Robins. var. altissima. Wallis listed villicaule Fern.

Ambrosia artemisiifolia L. var. elatior (L.)

Descourtils. Wallis listed forma villosa

Fern. \& Griseb.

Ambrosia bidentata Michx.

Ambrosia psilostachya DC. Syn. $=A$.

psilostachya DC. var. lindheimeriana

(Scheele) Blank.

Ambrosia trifida L. var. texana Scheele

Amphiachyris dracunculoides (DC.) Nutt. Syn. = Gutierrezia dracunculoides (DC.) Blake.

Antennaria neglecta Greene. Syn. $=A$. campestris Rydb.

\#Antennaria parlinii Fern.

\#Antennaria parlinii Fern. ssp. fallax (Greene)

Bayer \& Stebbins

Antennaria plantaginifolia (L.) Richards

*Anthemis cotula L.

Aphanostephus skirrhobasis (DC.) Trel.

${ }^{*}$ Arctium minus (Hill) Bernh.

Arnoglossum atriplicifolium (L.) H.E. Robins. 
Syn. = Cacalia atriplicifolia L .

Arnoglossum plantagineum Raf. Syn. =

Cacalia plantaginea (Raf.) Shinners.

Arnoglossum reniforme (Hook.) H.E. Robins.

Syn. = Cacalia muehlenbergii (Schultz-

Bip.) Fern.

${ }^{*}$ Artemisia annua $\mathrm{L}$.

Artemisia ludoviciana Nutt. ssp. Iudoviciana.

Syn. $=$ A. Iudoviciana Nutt. var.

gnaphalodes (Nutt.) Torr. \& Gray

Artemisia ludoviciana Nutt. ssp. mexicana

(Willd. ex Spreng.) Keck. Syn. = Artemisia

ludoviciana Nutt. var. mexicana (Willd. ex

Spreng.) Gray

Astranthium integrifolium (Michx.) Nutt.

\#Baccharis halimifolia L.

Berlandiera pumila (Michx.) Nutt. var. pumila.

Syn. = B. tomentosa Nutt. var. dealbata

Torr. \& Gray.

Bidens aristosa (Michx.) Britt. Syns. $=B$.

polylepis Blake var. polylepis and $B$.

polylepis Blake var. retrorsa Sherff.

Bidens bipinnata L.

\#Bidens cernua L.

\#Bidens discoidea (Torr. \& Gray) Britt.

Bidens frondosa $\mathrm{L}$.

Boltonia asteroides (L.) L'Hér. var. latisquama

(Gray) Cronq. Syn. = B. latisquama Gray.

Boltonia diffusa Ell. var. interior Fern. \& Grisc.

Brickellia eupatorioides (L.) Shinners var.

texana (Shinners) Shinners. Syn. =

Kuhnia eupatoriodes L. var. ozarkana

Shinners.

${ }^{*}$ Carduus nutans L.

Centaurea americana Nutt.

*Centaurea cyanus L.

Chaetopappa asteroides Nutt. ex DC.

Chrysopsis pilosa Nutt. Syn. = Heterotheca

pilosa (Nutt.) Shinners

*Cichorium intybus $\mathrm{L}$.

Cirsium altissimum (L.) Hill

${ }^{*}$ Cirsium vulgare (Savi) Ten.

\#Cirsium undulatum (Nutt.) Spreng.

Conoclinium coelestinum (L.) DC. Syn. =

Eupatorium coelestinum $\mathrm{L}$.

Conyza canadensis (L.) Cronq. var. canadensis
Conyza canadensis (L.) Cronq. var. glabrata (Gray) Cronq.

Coreopsis grandiflora Hogg ex Sweet var. grandiflora

\#Coreopsis grandiflora Hogg ex Sweet var. harveyana (Gray) Sherff

Coreopsis lanceolata L. Syn. = C. lanceolata

L. var. villosa Michx.

Coreopsis palmata Nutt.

Coreopsis pubescens Ell. var. pubescens

Coreopsis tinctoria Nutt. Wallis listed formas tinctoria and atropurpurea (Hook) Fern.

Coreopsis tripteris L. Syn. = C. tripteris L. var. deamii Standl.

*\#Cosmos sulphureus Cav.

*\#Crepis pulchra L.

Dracopis amplexicaulis (Vahl) Cass.

\#Echinacea angustifolia DC.

\#Echinacea atrorubens Nutt.

Echinacea pallida (Nutt.) Nutt.

Echinacea purpurea (L.) Moench

Eclipta alba (L.) L.

Elephantopus carolinianus Raeusch.

Erechtites hieraciifolia (L.) Raf. ex DC. var. hieraciifolia. Syns. $=E$. hieraciifolia $(\mathrm{L}$.

Raf. ex DC. var. intermedia Fern. and $E$. hieraciifolia (L.) Raf. ex DC. var. praealta (Raf.) Fern.

Erigeron annuus (L.) Pers.

Erigeron philadelphicus L. var. philadelphicus

Erigeron pulchellus Michx.

Erigeron strigosus Muhl. ex Willd. var. beyrichii (Fisch. \& C.A. Mey.) Torr. \& Gray ex Gray

Erigeron strigosus Muhl. ex Willd. var. strigosus

\#Erigeron tenuis Torr. \& Gray

Eupatorium altissimum L.

\#Eupatorium hyssopifolium L.

Eupatorium perfoliatum L.

Eupatorium purpureum L.

Eupatorium serotinum Michx.

\#Eupatoriadelphus fistulosus (Barratt) King \&

H.E. Robins.

Eurybia hemispherica (Alexander) Nesom.

Syn. = Aster hemisphericus Alexander

Euthamia gymnospermoides Greene. Syn. =

Solidago gymnospermoides (Greene)Fern.

Hoagland, B.W. 
Oklahoma Native Plant Record

Volume 7, Number 1, December 2007

Fleischmannia incarnata (Walt.) King \& H.E. Robins. Syn. $=$ Eupatorium incarnatum Walt.

Gaillardia aestivalis (Walt.) H. Rock var. aestivalis. Syn. $=$ G. fastigiata Greene.

Gaillardia aestivalis (Walt.) H. Rock var. flavovirens (C. Mohr) Cronq. Syn. = G. lutea Greene.

\#Gaillardia suavis (Gray \& Engelm.) Britt. \& Rusby

${ }^{*}$ Galinsoga parviflora Cay.

${ }^{*}$ \#Galinsoga quadriradiata Cav.

Gamochaeta purpurea (L.) Cabrera. Syn. = Gnaphalium purpureum L.

Grindelia lanceolata Nutt. var. lanceolata. Wallis listed forma lanceolata.

Grindelia papposa Nesom \& Suh. Syn. = Haplopappus ciliatus (Nutt.) DC.

Helenium amarum (Raf.) H. Rock

Helenium autumnale $\mathrm{L}$.

Helenium flexuosum Raf.

Helianthus angustifolius $\mathrm{L}$.

Helianthus annuus L.

\#Helianthus decapetalus $\mathrm{L}$.

\#Helianthus divaricatus $\mathrm{L}$.

Helianthus $\times$ doronicoides Lam. (pro sp.)

[giganteus $\times$ mollis]. Syn. $=H$.

doronicoides Lam.

Helianthus grosseserratus Martens

Helianthus hirsutus Raf. Syns. $=H$. hirsutus

Raf. var. stenophyllus Torr. \& Gray and $H$. hirsutus Raf. var. trachyphyllus Torr. \&

Gray.

\#Helianthus xlaetiflorus Pers. (pro sp.)

[pauciflorus $\times$ tuberosus]

Helianthus maximiliani Schrad.

Helianthus mollis Lam.

\#Helianthus nuttallii Torr. \& Gray

\#Helianthus laetiflorus Pers. var. rigidus

(Cass.) Fern.

Helianthus petiolaris Nutt.

Helianthus salicifolius A. Dietr.

Helianthus tuberosus L.

Heliopsis helianthoides (L.) Sweet var. scabra (Dunal) Fern.

Heterotheca subaxillaris (Lam.) Britt. \& Rusby. Syn. $=H$. latifolia Buckl.
Hieracium gronovii $\mathrm{L}$.

Hieracium longipilum Torr.

\#Hieracium scabrum Michx.

Hymenopappus scabiosaeus L'Hér. var. corymbosus (Torr. \& Gray) B.L. Turner

Hymenopappus scabiosaeus L'Hér. var. scabiosaeus

Ionactis linariifolius (L.) Greene. Syn. = Aster linarifolius $\mathrm{L}$.

Iva angustifolia Nutt. ex DC.

Iva annua L. var. annua. Syn. = I. ciliata Willd.

Krigia biflora (Walt.) Blake. Wallis listed formas biflora and glandulifera Fern.

Krigia dandelion (L.) Nutt.

Krigia caespitosa (Raf.) Chambers. Syn. = Serinia oppositifolia (Rat.) Kuntze

Krigia occidentalis Nutt.

Krigia virginica (L.) Willd.

Lactuca canadensis L. Syn. $=$ L. canadensis var. canadensis (Wallis listed formas angustata Wieg. and canadensis), $L$. canadensis L. var. latifolia Kuntze (Wallis listed formas latifolia and exauriculata Wieg.), L. canadensis L. var. longifolia (Michx.) Farw., and L. canadensis L. var. obovata Wieg. (Wallis listed forma stenopoda Wieg.).

Lactuca floridana (L.) Gaertn.

Lactuca Iudoviciana (Nutt.) Riddell. Wallis listed formas campestris (Greene) Fern. and ludoviciana.

*Lactuca serriola L. Syn. = L. scariola L. Wallis listed formas integrifolia (Bogenh.) G. Beck and scariola.

\#Lactuca tatarica (L.) C.A. Mey. var. pulchella (Pursh) Breitung

*Leucanthemum vulgare Lam. Syn. = Chrysanthemum leucanthemum L. var. pinnatifidum Lecoq \& Lamotte.

Liatris aspera Michx. var. aspera. Wallis listed formas aspera and benkii (Macbr.) Fern.

Liatris aspera Michx. var. intermedia (Lunell) Gaiser

\#Liatris punctata Hook.

\#Liatris punctata Hook. var. nebraskana Gaiser Liatris pycnostachya Michx. Wallis listed forma pycnostachya. 
Liatris squarrosa (L.) Michx. var. hirsuta (Rydb.) Gaiser

\#Liatris squarrosa (L.) Michx. var. glabrata (Rydb.) Gaiser

\#Liatris squarrulosa Michx.

*\#Matricaria discoidea DC.

Oligoneuron nitidum (Torr. \& Gray) Small. Syn. = Solidago nitida Torr. \& Gray.

\#Oligoneuron rigidum (L.) Small

Packera aurea (L.) A.\& D. Löve. Syn. = Senecio aureus $L$.

Packera glabella (Poir.) C. Jeffrey. Syn. = Senecio glabellus Poir.

Packera obovata (Muhl. ex Willd.) W.A. Weber $\&$ A. Löve. Syn. = Senecio obovatus Muhl var. rotundus Britt.

Packera plattensis (Nutt.) W.A. Weber \& A. Löve. Syn. = Senecio plattensis Nutt.

*\#Parthenium hysterophorus L.

Parthenium integrifolium $\mathrm{L}$.

Pluchea camphorata (L.) DC.

\#Pluchea odorata (L.) Cass. var. odorata

Polymnia canadensis $\mathrm{L}$. Wallis listed forma radiata (Gray) Fassett.

Prenanthes aspera Michx.

\#Prenanthes altissima $\mathrm{L}$.

Pseudognaphalium obtusifolium (L.) Hilliard \&

Burtt ssp. obtusifolium. Syn. =

Gnaphalium obtusifolium L.

Pyrrhopappus carolinianus (Walt.) DC.

\#Pyrrhopappus grandiflorus (Nutt.) Nutt.

\#Pyrrhopappus pauciflorus (D. Don) DC.

Ratibida columnifera (Nutt.) Woot. \& Standl.

Wallis listed formas columnifera and

pulcherrima (DC.) Fern.

Ratibida pinnata (Vent.) Barnh.

Rudbeckia grandiflora (D. Don) J.F. Gmel. ex

DC.

Rudbeckia hirta L. var. pulcherrima Farw.

Rudbeckia laciniata L. var. laciniata

Rudbeckia subtomentosa Pursh

Rudbeckia triloba L. var. triloba

\#Silphium asteriscus L.

\#Silphium integrifolium Michx. var. integrifolium

Silphium integrifolium Michx. var. laeve Torr. \&

Gray. Syn. = S. speciosum Nutt.

Silphium laciniatum L. var. laciniatum
Silphium perfoliatum $\mathrm{L}$.

Silphium radula Nutt. Syn. $=$ S. asperrimum Hook.

Smallanthus uvedalius (L.) Mackenzie ex Small. Syn. = Polymnia Uvedalia L. var. densipilis Blake

Solidago altissima L.

\#Solidago arguta Ait. var. boottii (Hook.) Palmer \& Steyermark

Solidago caesia L.

Solidago canadensis L. var. gilvocanescens Rydb.

Solidago gigantea Ait. Syn. = S. gigantea Ait. var. leiophylla Fern.

Solidago hispida Muhl. ex Willd.

Solidago ludoviciana (Gray) Small

Solidago missouriensis Nutt. var. fasciculata Holz.

Solidago nemoralis Ait. var. longipetiolata (Mackenzie \& Bush) Palmer \& Steyermark. Syn. = S. nemoralis Ait. var. decemflora (DC.) Fern.

Solidago nemoralis Ait. var. nemoralis. Syn. = S. nemoralis Ait. var. haleana Fern.

\#Solidago odora Ait.

Solidago petiolaris Ait. var. angusta (Torr. \& Gray) Gray. Syns. = S. lindheimeriana Scheele and S. petiolaris Ait. var. wardii (Britt.) Fern.

Solidago radula Nutt. var. radula

Solidago rugosa P. Mill. ssp. aspera (Ait.)

Cronq. Syn. = S. rugosa Mill. var. celtidifolia (Small) Fern.

Solidago speciosa Nutt. var. speciosa

\#Solidago speciosa Nutt. var. rigidiuscula Torr. \& Gray

Solidago ulmifolia Muhl. ex Willd. var. ulmifolia

Solidago ulmifolia Muhl. ex Willd. var. microphylla Gray. Syn. = S. delicatula Small.

*Sonchus asper (L.) Hill. Wallis listed forma glandulosus Beckh.

Symphyotrichum anomalum (Engelm.) Nesom. Syn. = Aster anomalus Engelm.

Symphyotrichum cordifolium (L.) Nesom. Syn. = Aster sagittifolius Wedemeyer ex Willd. var. sagittifolius. 
Symphyotrichum divaricatum (Nutt.) Nesom. Syn. = Aster exilis Ell.

Symphyotrichum drummondii (Lindl.) Nesom var. drummondii. Syn. = Aster sagittifolius Wedemeyer ex Willd. var. drummondii (Lindl.) Shinners.

Symphyotrichum ericoides (L.) Nesom var. ericoides. Syn. = Aster ericoides $\mathrm{L}$.

Symphyotrichum laeve (L.) A.\& D. Löve var. laeve. Syn. = Aster laevis L.

\#Symphyotrichum lanceolatum (Willd.) Nesom Symphyotrichum novae-angliae (L.) Nesom. Syn. = Aster novae-angliae $\mathrm{L}$.

Symphyotrichum oblongifolium (Nutt.) Nesom. Syn. $=$ Aster oblongifolius Nutt.

Symphyotrichum ontarionis (Wieg.) Nesom. Syn. = Aster ontarionis Wieg.

Symphyotrichum oolentangiense (Riddell) Nesom var. oolentangiense. Syn. = Aster azureus Lindl. var. azureus.

Symphyotrichum oolentangiense (Riddell) Nesom var. poaceum (Burgess) Nesom. Syn. = Aster azureus Lindl. var. poaceus (Burgess) Fern.

Symphyotrichum patens (Ait.) Nesom var. gracile (Hook.) Nesom. Syn. = Aster patens Ait. var. gracilis Hook.

Symphyotrichum patens (Ait.) Nesom var. patens. Syn. $=$ Aster patens Ait. var. patens.

Symphyotrichum pilosum (Willd.) Nesom. Syn. $=$ Aster pilosus Willd.

Symphyotrichum praealtum (Poir.) Nesom var. praealtum. Syn. $=$ Aster praealtus Poir. var. praealtus.

\#Symphyotrichum subulatum (Michx.) Nesom. Symphyotrichum turbinellum (Lindl.) Nesom.

Syn. = Aster turbinellus Lindl.

*\#Tanacetum vulgare L.

*Taraxacum officinale G.H. Weber ex Wiggers ssp. officinale

\#Thelesperma ambiguum Gray

${ }^{*}$ Tragopogon dubius Scop. Syn. = T. major Jacq.

Verbesina alternifolia (L.) Britt. ex Kearney. Syn. = Actinomeris alternifolia (L.) DC.
\#Verbesina encelioides (Cav.) Benth. \& Hook. f. ex Gray

Verbesina helianthoides Michx.

Verbesina virginica $\mathrm{L}$.

Vernonia arkansana DC. Syn. = V. crinita Raf. Vernonia baldwinii Torr. ssp. baldwinii

Vernonia gigantea (Walt.) Trel. ssp. gigantea.

Syn. $=V$. altissima Nutt.

Vernonia missurica Raf.

Xanthium strumarium $L$. var. canadense ( $P$.

Mill.) Torr. \& Gray. Syns. $=X$. italicum

Mor., $X$. pensylvanicum Wallr., and $X$. speciosum Kearney.

Xanthium strumarium L. var. glabratum (DC.) Cronq. Syn. $=X$. chinense Mill.

\section{Balsaminaceae}

Impatiens capensis Neerb.

Impatiens pallida Nutt.

Podophyllum peltatum $\mathrm{L}$.

\section{Berberidaceae}

Betulaceae (=Corylaceae)

Alnus serrulata (Alt.) Willd. Syn. = A. serrulata (Alt.) Willd. var. vulgaris Spach.

Betula nigra L.

Corylus americana Walt. var. americana. Wallis listed forma americana.

Ostrya virginiana (P. Mill.) K. Koch var. virginiana. Syn. = Ostrya virginiana $(P$. Mill.) K. Koch var. lasia Fern. Wallis listed forma glandulosa (Spach) Macbr.

\section{Bignoniaceae}

Campsis radicans (L.) Seem.

Catalpa bignonioides Walt.

Catalpa speciosa (Warder) Warder ex Engelm.

\section{Boraginaceae}

${ }^{*}$ Buglossoides arvensis (L.) I.M. Johnston.

Syn. = Lithospermum arvense L.

\#Cynoglossum virginianum $L$.

Hackelia virginiana (L.) I.M. Johnston

Heliotropium convolvulaceum (Nutt.) Gray

*Heliotropium indicum L.

Heliotropium tenellum (Nutt.) Torr. 
Lithospermum canescens (Michx.) Lehm.

Lithospermum caroliniense (Walt. ex J.F.

Gmel.) MacM.

Lithospermum incisum Lehm.

Myosotis macrosperma Engelm.

Myosotis verna Nutt.

Onosmodium bejariense DC. ex A. DC. var. occidentale (Mackenzie) B.L. Turner. Syn. $=0$. occidentale Mackenzie.

Onosmodium bejariense DC. ex A. DC. var. subsetosum (Mackenzie \& Bush) B.L. Turner. Syn. $=0$. subsetosum Mackenzie \& Bush.

\section{Brassicaceae}

${ }^{*}$ \#Alliaria petiolata (Bieb.) Cavara \& Grande Arabis canadensis L.

Arabis laevigata (Muhl. ex Willd.) Poir.

Arabis missouriensis Greene

Arabis shortii (Fern.) Gleason. Syn. = A.

perstellata E.L. Br. var. shortii Fern.

${ }^{*}$ Barbarea vulgaris Ait. f.

*Brassica napus L.

*Brassica rapa L.

*Camelina microcarpa Andrz. ex DC.

${ }^{*}$ Capsella bursa-pastoris (L.) Medik.

Cardamine bulbosa (Schreb. ex Muhl.) B.S.P.

Cardamine parviflora L. var. arenicola (Britt)

O.E. Shultz

Cardamine pensylvanica Muhl. ex Willd.

Cardamine concatenata (Michx.) Sw. Syn. = Dentaria laciniata Muhl.

Descurainia pinnata (Walt.) Britt. ssp. brachycarpa (Richards.) Detling

Draba aprica Beadle

Draba brachycarpa Nutt. ex Torr. \& Gray

Draba cuneifolia Nutt. ex Torr. \& Gray var. cuneifolia

\#Draba reptans (Lam.) Fern.

lodanthus pinnatifidus (Michx.). Steud.

Lepidium campestre (L.) Ait. f.

Lepidium densiflorum Schrad.

Lepidium virginicum L. var. virginicum

Lesquerella gracilis (Hook.) S. Wats. var. gracilis

*Nasturtium officinale Ait. f.

Neobeckia aquatica (Eat.) Greene. Syn. =
Armoracia aquatica (Eat.) Wieg.

Rorippa palustris (L.) Bess. ssp. fernaldiana

(Butters \& Abbe) Jonsell. Syn. $=R$.

islandica (Oeder) Borbas var. fernaldia Butters \& Abbe.

Rorippa palustris (L.) Bess. ssp. hispida

(Desv.) Jonsell. Syn. $=R$. islandica

(Oeder) Borbas var. hispida (Desv.)

Butters \& Abbe.

Rorippa teres (Michx.) R. Stuckey. Syn. $=R$. obtusa (Nutt.) Britt.

Rorippa sessiliflora (Nutt.) A.S. Hitchc.

Selenia aurea Nutt.

Sibara virginica (L.) Rollins

*Sinapis arvensis L. Syn. = Brassica kaber

(DC.) L.C. Wheeler var. pinnatifida

(Stokes) L.C. Wheeler.

*\#Sisymbrium altissimum L.

*Sisymbrium officinale (L.) Scop. Syn. = S.

officinale (L.) Scop. var. leiocarpum DC.

Streptanthus maculatus Nutt.

*Thlaspi arvense L.

\section{Cabombaceae}

\#Brasenia schreberi J.F. Gmel.

\section{Cactaceae}

\#Opuntia humifusa (Raf.) Raf.

Opuntia macrorhiza Engelm.

Callitrichaceae

Callitriche heterophylla Pursh

Callitriche terrestris Raf.

\section{Campanulaceae}

Campanulastrum americanum (L.) Small. Syn. = Campanula americana L. var. illinoensis (Fresn.) Farw.

Lobelia appendiculata A. DC.

\#Lobelia puberula Michx.

Lobelia cardinalis L.

Lobelia inflata L.

Lobelia siphilitica L. var. Iudoviciana A. DC.

Lobelia spicata Lam. var. spicata

Lobelia spicata Lam. var. leptostachys (A. DC.) Mackenzie \& Bush

Triodanis biflora (Ruiz \& Pavón) Greene. Syn. 
Oklahoma Native Plant Record

Volume 7, Number 1, December 2007

= Specularia biflora (Ruiz \& Pavón) Fisch.

\& C.A. Mey.

Triodanis lamprosperma McVaugh. Syn. = Specularia lamprosperma (McVaugh) Fern.

Triodanis leptocarpa (Nutt.) Nieuwl. Syn. = Specularia leptocarpa (Nutt.) Gray

Triodanis perfoliata $(\mathrm{L}$.) Nieuwl. Syn. = Specularia perfoliata (L.) A. DC.

*Humulus lupulus L.

\section{Cannabinaceae}

\section{Capparidaceae}

*\#Cleome hassleriana Chod.

\#Cleome serrulata Pursh

\#Polanisia dodecandra (L.) DC. ssp. dodecandra

Polanisia dodecandra (L.) DC. ssp. trachysperma (Torr. \& Gray) Iltis

Lonicera flava Sims

\section{Caprifoliaceae}

*Lonicera japonica Thunb.

\#Lonicera sempervirens L.

Sambucus nigra L. ssp. canadensis (L.) R.

Bolli. Syn. = S. canadensis L. var.

canadensis and $S$. canadensis L. var.

submollis Rehd.

Symphoricarpos orbiculatus Moench.

\# Triosteum aurantiacum Bickn.

Triosteum perfoliatum $\mathrm{L}$.

\#Viburnum molle Michx.

\#Viburnum rafinesquianum J.A. Schultes

Viburnum rufidulum Raf.

Agrostemma githago L.

\section{Caryophyllaceae}

*Arenaria serpyllifolia L.

Cerastium brachypodum (Engelm. ex Gray)

B.L. Robins.

\#Cerastium brachypetalum Desportes ex Pers.

${ }^{*}$ Cerastium fontanum Baumg. ssp. vulgare

(Hartman) Greuter \& Burdet. Syn. = C. vulgatum $\mathrm{L}$.

${ }^{*}$ Cerastium glomeratum Thuill. Syn. $=$ C . viscosum auct. non $\mathrm{L}$.

Cerastium nutans Raf.
*\#Cerastium pumilum W. Curtis

${ }^{*}$ Dianthus armeria L.

Minuartia drummondii (Shinners) McNeill. Syn. = Stellaria nuttallii Torr. \& Gray.

\#Minuartia michauxii (Fenzl) Farw. var. texana (B.L. Robins.) Mattf.

Minuartia muscorum (Fassett) Rabeler. Syn. = Stellaria muscorum Fassett.

Minuartia patula (Michx.) Mattf. Syn. = Arenaria patula Michx. Wallis listed formas media Steyerm. pitcheri (Nutt.) Steyerm. and robusta Steyerm.

Paronychia canadensis (L.) Wood

Paronychia fastigiata (Raf.) Fern. var. fastigiata

*\#Petrorhagia dubia (Raf.) G. López \& Romo

*Petrorhagia prolifera (L.) P.W. Ball \&

Heywood. Syn. = Dianthus prolifer $\mathrm{L}$.

Sagina decumbens (Ell.) Torr. \& Gray

*Saponaria officinalis L.

*\#Scleranthus annuus L.

Silene antirrhina L.

Silene regia Sims

Silene stellata (L.) Ait. f. Wallis listed forma scabrella (Niewl.) Palm. \& Steyerm.

Silene virginica $\mathrm{L}$.

*Stellaria media (L.) Vill.

\section{Celastraceae}

\#Celastrus scandens L.

Euonymus atropurpureus Jacq.

\section{Ceratophyllaceae \\ Ceratophyllum demersum $\mathrm{L}$.}

\section{Chenopodiaceae}

Chenopodium album L.

${ }^{*}$ Chenopodium ambrosioides L. var. ambrosioides

\#Chenopodium berlandieri Moq.

Chenopodium leptophyllum (Moq.) Nutt. ex S. Wats.

${ }^{*}$ Chenopodium pumilio R. Br.

Chenopodium simplex (Torr.) Raf. Syn. $=C$. hybridum L. var. gigantospermum (Aellen) Rouleau.

Chenopodium standleyanum Aellen Cycloloma atriplicifolium (Spreng.) Coult. 
Monolepis nuttalliana (J.A. Schultes) Greene

\section{Cistaceae}

Lechea mucronata Raf. Syn. = L. villosa Ell.

Lechea tenuifolia Michx. Syn. = Lechea

tenuifolia Michx.var. occidentalis Hodgdon.

Clusiaceae (= Guttiferae)

Hypericum hypericoides (L.) Crantz ssp.

hypericoides. Syn. = Ascyrum

hypericoides L. var. hypericoides.

Hypericum hypericoides (L.) Crantz ssp.

multicaule (Michx. ex Willd.) Robson. Syn.

= Ascyrum hypericoides L. var. multicaule

(Michx.) Fern.

Hypericum drummondii (Grev. \& Hook.) Torr. \&

Gray

Hypericum gentianoides (L.) B.S.P.

Hypericum multilum L. Syn. $=H$. multilum L.

var. parviflorum (Willd.) Fern.

*Hypericum perforatum L.

Hypericum pseudomaculatum Bush

Hypericum prolificum L. Syn. $=H$.

spathulatum (Spach.) Steud.

Hypericum punctatum Lam.

Hypericum sphaerocarpum Michx.

\section{Convolvulaceae}

${ }^{*}$ Calystegia sepium (L.) R. Br. ssp. sepium

${ }^{*}$ Convolvulus arvensis $\mathrm{L}$. Syn. = C. arvensis

L. var. fraterniflorus Mack. \& Bush.

*\#lpomoea coccinea $\mathrm{L}$.

\#lpomoea pandurata (L.) G.F.W. Mey.

*\#lpomoea purpurea (L.) Roth

\#Calystegia silvatica (Kit.) Griseb. ssp.

fraterniflora (Mackenzie \& Bush) Brummitt

*Ipomoea hederacea Jacq. Syn. $=$ I.

hederacea (L.) Jacq. var. integriuseula

Gray.

Ipomoea lacunosa $\mathrm{L}$.

Ipomoea pandurata (L.) G.F.W. Mey.

\section{Cornaceae}

Cornus drummondi C.A. Meyer

Cornus florida L.

Cornus obliqua Raf.

\section{Crassulaceae}

Sedum nuttallianum Raf.

Sedum pulchellum Michx.

*\#Sedum sarmentosum Bunge

Cucurbitaceae

\#Cayaponia grandifolia (Torr. \& Gray) Small

Cucurbita foetidissima Kunth

Melothria pendula L.

Sicyos angulatus $\mathrm{L}$.

\section{Cuscutaceae}

Cuscuta compacta Juss. ex Choisy

Cuscuta cuspidata Engelm.

Cuscuta glomerata Choisy

Cuscuta gronovii Willd. ex J.A. Schultes

\#Cuscuta indecora Choisy

\#Cuscuta obtusiflora Kunth

Cuscuta pentagona Engelm. var. pentagona.

Syn. $=C$. campestris Yuncker.

Cuscuta pentagona Engelm. var. glabrior

(Engelm.) Gandhi, Thomas \& Hatch. Syn.

= C. glabrior (Engelm.) Yuncker.

\#Cuscuta polygonorum Engelm.

\section{Dipsacaceae}

${ }^{*}$ Dipsacus fullonum L. Syn. = Dipsacus

sylvestris Huds.

\section{Droseraceae}

\#Drosera brevifolia Pursh

\section{Ebenaceae}

Diospyros virginiana $\mathrm{L}$. Syn. $=D$. virginiana $\mathrm{L}$. var. pubescens (Pursh) Dippel.

Elaeagnaceae
*\#Elaeagnus angustifolia L.

\section{Ericaceae}

Rhododendron canescens (Michx.) Sweet

\#Rhododendron oblongifolium (Small) Millais

\#Rhododendron prinophyllum (Small) Millais

Vaccinium arboreum Marsh. Syn. = Vaccinium

arboreum Marsh. var. glaucescens

(Greene) Sarg.

Vaccinium pallidum Ait. Syn. $=$ V. vacillans

Hoagland, B.W. 
Torr. var. crinitum Fern.

Vacciniun stamineum $L$. Syn. $=V$. stamineum

L. var. interius (Ashe) Palmer \& Steyerm.

and $V$. stamineum L. var. neglectum

(Small) Deam)

\#Vaccinium virgatum Ait.

\section{Euphorbiaceae}

Acalypha gracilens Gray. Syn. = A. gracilens

Gray var. fraseri (Muell.-Arg. Weatherby

Acalypha monococca (Engelm. ex Gray) L.

Mill. \& Gandhi. Syn. = A. gracilens Gray

var. monococca Engelm. ex Gray.

Acalypha ostryifolia Riddell

Acalypha rhomboidea Raf.

Acalypha virginica $\mathrm{L}$.

Argythamnia mercurialina (Nutt.) Muell.-Arg.

var. mercurialina. Syn. $=$ Ditaxis

mercurialina (Nutt.) Coult.

Chamaesyce humistrata (Engelm. ex Gray)

Small. Syn. $=$ Euphorbia humistrata

Engelm. ex Gray.

Chamaesyce maculata (L.) Small. Syn. =

Euphorbia maculata L. and Euphorbia supina Raf.

Chamaesyce missurica (Raf.) Shinners. Syn.

= Euphorbia missurica Raf.

\#Chamaesyce nutans (Lag.) Small

Chamaesyce prostrata (Ait.) Small. Syn. = Euphorbia chamaesyce auct. non L.

Chamaesyce serpens (Kunth) Small. Syn. = Euphorbia serpens Kunth.

Croton capitatus Michx. var. capitatus.

Croton glandulosus L. var. septentrionalis Muell.-Arg.

Croton lindheimerianus Scheele

\#Croton michauxii G.L. Webster

Croton monanthogynus Michx.

Croton willdenowii G.L. Webster. Syn. =

Crotonopsis elliptica Willd.

Euphorbia corollata L. var. paniculata (Ell.)

Boiss.

Euphorbia cyathophora Murr. Syn. = E. heterophylla L. var. graminifolia (Michx.) Engelm.

Euphorbia dentata Michx. Wallis listed formas cuphosperma (Engelm.) Fern. and dentata.
Euphorbia heterophylla L.

Euphorbia hexagona Nutt. ex Spreng.

Euphorbia marginata Pursh

Euphorbia pubentissima Michx. Syn. = E. corollata L. var. mollis Millap.

Euphorbia spathulata Lam. Syn. $=E$. dictyosperma Fisch. \& C.A. Mey.

Euphorbia obtusata (Pursh) Small

Phyllanthus caroliniensis Walt.

Stillingia sylvatica L.

Tragia betonicifolia Nutt. Syn. $=T$. urticifolia Michx.

\#Tragia urticifolia Michx.

Tragia ramosa Torr.

Fabaceae (=Leguminosae)

Acacia angustissima (P. Mill.) Kuntze var. hirta (Nutt.) B.L. Robins.

*\#Albizia julibrissin Durazz.

Amorpha canescens Pursh

Amorpha fruticosa $\mathrm{L}$. Syn. = A. fruticosa $\mathrm{L}$. var. angustifolia Pursh, $A$. fruticosa $L$. var. oblongifolia Palmer and $A$. fruticosa $\mathrm{L}$. var. tennesseensis (Shuttlew.) Palmer.

\#Amorpha laevigata Nutt.

Amphicarpaea bracteata (L.) Fern. var. bracteata

Amphicarpaea bracteata (L.) Fern. var. comosa (L.) Fern.

Apios americana Medik. Syn. = A. americana Medik. var. turrigera Fern.

Astragalus canadensis L.

Astragalus crassicarpus Nutt. var. trichocalyx (Nutt.) Barneby

Astragalus distortus Torr. \& Gray

\#Astragalus nuttallianus DC.

Baptisia alba (L.) Vent. var. macrophylla (Larisey) Isely. Syn. = Baptisia leucantha Torr. \& Gray var. leucantha.

Baptisia australis (L.) R. Br. ex Ait. f. var. minor (Lehm.) Fern.

\#Baptisia bracteata Muhl. ex Ell. var. leucophaea (Nutt.) Kartesz \& Gandhi

Cercis canadensis L. var. canadensis. Wallis listed formas canadensis and glabrifolia Fern.

Chamaecrista fasciculata (Michx.) Greene var. 
fasciculata. Syn. = Cassia fasiculata Michx. var. robusta (Pollard) Macbr.

Chamaecrista nictitans (L.) Moench ssp. nictitans var. nictitans. Syn. $=$ Cassia nictitans $\mathrm{L}$.

Cladrastis kentukea (Dum.-Cours.) Rudd. Clitoria mariana L.

Crotalaria sagittalis L.

Dalea candida Michx. ex Willd. var. candida

Dalea lanata Spreng

\#Dalea multiflora (Nutt.) Shinners

Dalea purpurea Vent.

Desmanthus illinoensis (Michx.) MacM. ex B.L. Robins. \& Fern.

Desmodium canadense (L.) DC.

Desmodium canescens (L.) DC.

Desmodium ciliare (Muhl. ex Willd.) DC.

Desmodium cuspidatum (Muhl. ex Willd.) DC. ex Loud.

Desmodium glutinosum (Muhl. ex Willd.) Wood

\#Desmodium illinoense Gray

Desmodium laevigatum (Nutt.) DC.

Desmodium marilandicum (L.) DC.

Desmodium nudiflorum (L.) DC. Wallis lists formas foliolatum (Farwell) Fassett, nudiflorum, and personatum Fassett.

\#Desmodium nuttallii (Schindl.) Schub.

Desmodium obtusum (Muhl. ex Willd.) DC. Syn. = Desmodlum rigidum (Ell.) DC.

Desmodium paniculatum (L.) DC. var. paniculatum

Desmodium pauciflorum (Nutt.) DC.

Desmodium perplexum Schub. Syn. = Desmodium paniculatum (L.) DC. var. dillenii (Darl.) Isely.

Desmodium rotundifolium DC.

Desmodium sessilifolium (Torr.) Torr. \& Gray \#Desmodium viridiflorum (L.) DC.

Galactia volubilis (L.) Britt. Syn. = G. volubilis

(L.) Britt. var. mississippiensis Vail.

Gleditsia triacanthos L.

Gymnocladus dioicus (L.) K. Koch

\#Indigofera miniata Ortega

*Kummerowia stipulacea (Maxim.) Makino

*Kummerowia striata (Thunb.) Schindl.

*\#Lathyrus hirsutus L.

*Lathyrus latifolius L.
Lathyrus pusillus Ell.

Lespedeza capitata Michx.

Lespedeza cuneata (Dum.-Cours.) G. Don

\#Lespedeza frutescens (L.) Hornem.

Lespedeza hirta (L.) Hornem.

Lespedeza procumbens Michx.

Lespedeza repens (L.) W. Bart.

Lespedeza stuevei Nutt.

*\#Lespedeza thunbergii (DC.)Nakai

Lespedeza violacea (L.) Pers.

Lespedeza virginica (L.) Britt.

Lotus unifoliolatus (Hook.) Benth. var.

unifoliolatus. Syn. = L. americanus (Mitt.)

Bisch. non Vell.

*Medicago lupulina L.

*Medicago sativa L.

${ }^{*}$ Melilotus officinalis (L.) Lam. Syn. = M. alba Medikus.

Mimosa nuttallii (DC.) B.L. Turner. Syn. = Schrankia nuttallii (DC.) Standl.

Neptunia lutea (Leavenworth) Benth.

Orbexilum pedunculatum (P. Mill.) Rydb. var. pedunculatum. Syn. $=$ Psoralea psoralioides (Walt.) Cory var. eglandulosa (Ell.) Freeman.

\#Orbexilum simplex (Nutt. ex Torr. \& Gray) Rydb.

\#Pediomelum linearifolium (Torr. \& Gray) J. Grimes

Phaseolus polystachios (L.) B.S.P.

Psoralidium tenuiflorum (Pursh) Rydb. Syn. = $P$. tenuiflora Pursh var. floribunda (Nutt.) Rydb.

*\#Pueraria montana (Lour.) Merr.

Rhynchosia latifolia Nutt. ex Torr. \& Gray

\#Robinia hispida L.

Robinia pseudo-acacia L.

Senna marilandica (L.) Link. Syn. = Cassia marilandica $\mathrm{L}$.

Sesbania herbacea (P. Mill.) McVaugh. Syn. = S. exaltata Raf.

Strophostyles helvula (L.) Elliot

Strophostyles leiosperma (Torr. \& Gray) Piper

Strophostyles umbellata (Muhl. ex Willd.) Britt.

Stylosanthes biflora (L.) B.S.P. Syn. = S. biflora (L.) B.S.P. var. hispidissima (Michx.) Pollard \& Ball. 
Oklahoma Native Plant Record

Volume 7, Number 1, December 2007

Tephrosia virginiana (L.) Pers. Syn. $=T$. virginiana (L.) Pers. var. holosericea (Nutt.) Torr. \& Gray.

${ }^{*}$ Trifolium arvense $\mathrm{L}$.

Trifolium carolinianum Michx.

*Trifolium dubium Sibthorp

*Trifolium hybridum L.

*Trifolium incarnatum $\mathrm{L}$.

*Trifolium pratense $\mathrm{L}$.

Trifolium campestre Schreb. Syn. $=T$. procumbens $\mathrm{L}$.

Trifolium reflexum $\mathrm{L}$.

${ }^{*}$ Trifolium repens $\mathrm{L}$.

${ }^{*}$ Trifolium resupinatum $\mathrm{L}$.

Vicia caroliniana Walt.

Vicia ludoviciana Nutt. ssp. leavenworthii (Torr.

\& Gray) Lassetter \& Gunn. Syn. = V. leavenworthii Torr. \& Gray var. leavenworthii.

\#Vicia ludoviciana Nutt. ssp. Iudoviciana

Vicia minutiflora F.G. Dietr.

*Vicia sativa L. ssp. nigra (L.) Ehrh. Syn. = V. angustifolia L. var. segetalis (Thuill.)

W.D.J. Koch.

*Vicia villosa Roth

\section{Fagaceae}

Castanea pumila (L.) P. Mill. var. ozarkensis

(Ashe) Tucker. Syn. = C. ozarkensis Ashe.

Quercus alba L. Wallis listed formas alba, latiloba (Sarg,) Palmer \& Steyerm. and viridis Trel.

\#Quercus buckleyi Nixon \& Dorr

Quercus falcata Michx. var. falcata

Quercus lyrata Walt.

Quercus macrocarpa Michx.

Quercus marilandica (L.) Muenchh.

Quercus muehlenbergii Engelm. Wallis lists forma alexanderi (Britt.) Trel.

Quercus nigra L. var. nigra

Quercus palustris Muenchh.

Quercus rubra L. var. ambigua (Gray) Fern.

Syn. $=Q$. rubra L. var. borealis (Michx. f.)

Farw.

Quercus rubra L. var. rubra

Quercus shumardii Buckl. var. schneckii (Britt.)
Quercus stellata Wangenh.

Quercus velutina Lam. Wallis listed formas dilaniata Thel., macrophylla (Dippel) Trel., and missouriensis (Sarg.) Trel.

\section{Fumariaceae}

\#Corydalis curvisiliqua Engelm. ssp. occidentalis (Engelm. ex Gray) W.A. Weber

Corydalis crystallina Engelm. Corydalis flavula (Raf.) DC.

Corydalis micrantha (Engelm. ex Gray) Gray

Dicentra cucullaria (L.) Bernh.

\section{Gentianaceae}

\#Gentiana alba Muhl. ex Nutt.

\#Gentiana puberulenta J. Pringle

Sabatia angularis (L.) Pursh

Sabatia campestris Nutt. Wallis listed formas albiflora D. M. Moore and campestris.

\section{Geraniaceae}

*Erodium cicutarium (L.) L'Hér. ex Ait.

Geranium carolinianum L.

\#Geranium carolinianum L. var. sphaerospermum (Fern.) Breitung

Geranium maculatum $\mathrm{L}$.

*\#Geranium molle L.

*Geranium pusillum L.

\#Ribes missouriense Nutt.

\section{Grossulariaceae}

Haloragaceae

*Myriophyllum aquaticum (Vell.) Verdc. Syn. = M. brasiliense Camb.

Myriophyllum heterophyllum Michx.

Myriophyllum pinnatum (Walt.) B.S.P.

\section{Hamamelidaceae}

Hamamelis vernalis Sarg. Syn. $=H$. vernalis

Sarg. var. tomentella (Rehd.) Palmer.

\section{Hippocastanaceae}

Aesculus glabra Willd. var. glabra. Syn. = A glabra Willd. var. sargentii Rehd.

Sarg. 


\section{Hydrangeaceae}

Hydrangea arborescens L. var. arborescens. Syn. $=H$. arborescens L. var. oblonga Torr. \& Gray

Hydrangea cinerea Small. Syn. $=H$.

arborescens L. var. deamii St. John.

Philadelphus pubescens Loisel.

\section{Hydrocharitaceae}

*\#Egeria densa Planch.

\#Elodea canadensis Michx.

Ellisia nyctelea (L.)L.

\section{Hydrophyllaceae}

Hydrolea ovata Nutt. ex Choisy

Hydrophyllum virginianum L.

Nemophila phacelioides Nutt.

\#Phacelia gilioides Brand

Phacelia hirsuta Nutt.

Phacelia strictiflora (Engelm. \& Gray) Gray var. robbinsii Constance

\section{Juglandaceae}

Carya alba (L.) Nutt. ex Ell. Syn. = Carya tomentosa (Lam. ex Poir.) Nutt.

Carya cordiformis (Wangenh.) K. Koch

\#Carya glabra (P. Mill.) Sweet

Carya illinoensis (Wangenh.) K. Koch

\#Carya laciniosa (Michx. f.) G. Don

Carya ovalis (Wangenh.) Sarg. Syn. = Carya

ovalis (Wangenh.) Sarg. var. obcordata

(Muhl. \& Willd.) Sarg.

Carya ovata (P. Mill.) K. Koch

Carya texana Buckl.

Juglans nigra L.

Lamiaceae (= Labiatae)

Agastache nepetoides (L.) Kuntze

\#Blephilia ciliata (L.) Benth.

Clinopodium arkansanum (Nutt.) House. Syn.

= Satureja arkansana (Nutt.) Briq.

Cunila origanoides (L.) Britt.

${ }^{*}$ Glechoma hederacea L. Syn. = G. hederacea

L. var. micrantha Moricand.

Hedeoma hispida Pursh

Hedeoma pulegioides (L.) Pers.

*Lamium amplexicaule L. Wallis listed formas albiflorum D. M. Moore and amplexicaule.

*Lamium purpureum L.

*Leonurus cardiaca L.

*Leonurus sibiricus L.

Lycopus americanus Muhl. ex W. Bart. Syn. =

L. americanus Muhl. var. scabrifolius Fern.

Lycopus rubellus Moench. Syn. = L. rubellus

Moench. var. arkansanus (Fresn.) Benner.

Lycopus uniflorus Michx.

*Marrubium vulgare L.

*Melissa officinalis L.

${ }^{*}$ Mentha xpiperita L. (pro sp.) [aquatica $\times$ spicata]. Syn. $=$ Mentha piperita $\mathrm{L}$.

*Mentha spicata L.

\#Monarda bradburiana Beck

Monarda citriodora Cerv. ex Lag.

Monarda fistulosa L. ssp. fistulosa

\#Monarda fistulosa L. ssp. fistulosa var. mollis (L.) Benth.

Monarda punctata L. ssp. punctata var. villicaulis (Pennell) Palmer \& Steyermark.

Syn. $=M$. punctata L. var. villicaulis

(Pennell) Shinners

Monarda russeliana Nutt. ex Sims. Syn. $=M$. virgata Raf.

*Nepeta cataria L.

${ }^{*}$ Perilla frutescens (L.) Britton

Physostegia angustifolia Fern.

\#Physostegia virginiana (L.) Benth.

Prunella vulgaris $L$. Syn. $=P$. caroliniana Mill.

\#Prunella vulgaris L. var. lanceolata (W. Bart.)

Fern.

Pycnanthemum albescens Torr. \& Gray

Pycnanthemum tenuifolium Schrad.

Pycnanthemum verticillatum (Michx.) Pers. var. pilosum (Nutt.) Cooperrider. Syn. $=P$.

pilosum Nutt.

Salvia azurea Michx. ex Lam. var. grandiflora Benth.

Salvia lyrata $\mathrm{L}$.

Scutellaria elliptica Muhl. ex Spreng.

Scutellaria incana Biehler

Scutellaria lateriflora L.

Scutellaria ovata Hill ssp. bracteata (Benth.)

Epling

Scutellaria ovata Hill ssp. ovata

Scutellaria parvula Michx. var. parvula 
Oklahoma Native Plant Record

Volume 7, Number 1, December 2007

\#Scutellaria parvula Michx. var. australis

Fassett

Stachys tenuifolia Willd.

Teucrium canadense L. var. canadense. Syn.

$=T$. canadense L. var. virginicum (L.) Eat.

Trichostema brachiatum L.

\section{Lauraceae}

Lindera benzoin (L.) Blume var. benzoin

Lindera benzoin (L.) Blume var. pubescens

(Palmer \& Steyerm.)

Rehd.

Sassafras albidum (Nutt.) Nees. Syn. = S. albidum (Nutt.) Nees var. molle (Raf.) Fern.

\section{Lentibulariaceae}

Utricularia gibba L. Syn. = U. biflora Lam.

\section{Linaceae}

\#Linum berlandieri Hook. var. berlandieri Hook. var. berlandieri

Linum medium (Planch.) Britt. var. texanum

(Planch.) Fern.

Linum pratense (J.B.S. Norton) Small. Syn. =

L. lewisii Pursh var. pratense J.B.S. Norton

Linum sulcatum Riddell

Loasaceae

Mentzelia oligosperma Nutt. ex Sims.

Polypremum procumbens L.

\section{Loganiaceae}

\section{Lythraceae}

Ammannia auriculata Willd.

Ammannia coccinea Rothb.

Cuphea viscosissima Jacq. Syn. $=$ C. petiolata

Koehne.

Lythrum alatum Pursh

Lythrum alatum Pursh var. lanceolatum (Ell.)

Torr. \& Gray ex Rothrock. Syn. = $L$.

lanceolatum Ell.

Rotala ramosior $(\mathrm{L}$.) Koehne. Syn. $=R$. ramosior (L.) Koehne var. interior Fern. \& Grisc.

Malvaceae

*Abutilon theophrasti Medik.
Callirhoe alcaeoides (Michx.) Gray

Callirhoe bushii Fern.

Callirhoe digitata Nutt. var. digitata

Callirhoe involucrata (Torr. and Gray) Gray var. involucrata

\#Callirhoe leiocarpa R.F. Martin

Hibiscus laevis All. Syn. $=H$. militaris Cav.

Hibiscus lasiocarpos Cav.

\#Hibiscus moscheutos L.

*\#Hibiscus syriacus L.

*\#Hibiscus trionum L.

*Malva neglecta Wallr.

Malvastrum hispidum (Pursh) Hochr. Syn. = Sidopsis hispida (Pursh) Rydb.

Sida spinosa L.

Glinus lotoides L.

Melastomataceae

Rhexia mariana L. var. interior (Pennell) Kral \& Bostick. Syn. $=R$. interior Pennell

\section{Menispermaceae}

Calycocarpum lyoni (Pursh) Gray

Cocculus carolinus (L.) DC.

Menispermum canadense $\mathrm{L}$.

Mollugo verticillata $L$.

\section{Molluginaceae}

\section{Monotropaceae}

\#Monotropa hypopithys L.

\#Monotropa uniflora $\mathrm{L}$.

\author{
Moraceae \\ Maclura pomifera (Raf.) Schneider \\ *Morus alba L. \\ Morus rubra L.
}

\section{Nelumbo lutea Willd.}

\section{Nelumbonaceae}

Mirabilis albida (Walt.) Heimerl Mirabilis linearis (Pursh) Heimerl *Mirabilis jalapa L.

Mirabilis nyctaginea (Michx.) MacM. 
Nymphaeaceae

Nuphar lutea (L.) Sm. ssp. advena (Ait.)

Kartesz \& Gandhi. Syn. = N. advena (Ait.)

Alt. $f$.

Nymphaea odorata Ait. ssp. tuberosa (Paine)

Wiersma \& Hellquist. Syn. $=$ N. tuberosa

Ait.

Nyssaceae

Nyssa sylvatica Marsh. Syn. = N. sylvatica

Marsh. var. dilatata Fern.

\section{Oleaceae}

\#Forestiera acuminata (Michx.) Poir.

Fraxinus americana L.

Fraxinus pennsylvanica Marsh. Syn. =

Fraxinus pennsylvanica Marsh. var.

subintegerrima (Vahl) Fern.

Fraxinus quadrangulata Michx.

*Ligustrum sinense Lour.

\section{Onagraceae}

\#Calylophus serrulatus (Nutt.) Raven

Circaea lutetiana L. ssp. canadensis (L.)

Aschers. \& Magnus. Syn. $=C$.

quadrisulcata (Maxim.) Franch. \& Savigny

ssp. canadensis (L.) A.\& D. Löve.

\#Gaura biennis L.

Gaura longiflora Spach. Syn. = G. biennis L.

var. pitcheri Torr. \& Gray.

Gaura mollis James. Syn. = G. parviflora Dougl. ex Lehm. var. parviflora. Wallis listed forma parviflora.

Ludwigia alternifolia L. Syn. = Ludwigia alternifolia L. var. pubescens Palmer \& Steyermark.

Ludwigia decurrens Walt. Syn. = Jussiaea decurrens (Walt.) DC.

Ludwigia glandulosa Walt. ssp. glandulosa

Ludwigia palustris (L.) Ell. Syn. = L. palustris

(L.) Ell. var. americana (DC.) Fern. \& Grisc.

Ludwigia peploides (Kunth) Raven ssp. glabrescens (Kuntze) Raven. Syn. = Jussiaea repens L. var. glabrescens Kuntze.

\#Ludwigia repens J.R. Forst.

Oenothera biennis L. var. biennis

Oenothera elata Kunth ssp. hirsutissima (Gray ex S. Wats.) W. Dietr. Syn. = O. biennis L. var. hirsutissima Gray.

Oenothera fruticosa L. ssp. fruticosa

\#Oenothera grandis (Britt.) Smyth

Oenothera laciniata Hill

Oenothera linifolia Nutt.

\#Oenothera spachiana Torr. \& Gray

\#Oenothera speciosa Nutt.

Oenothera villosa Thunb. ssp. villosa. Syn. =

O. biennis L. var. canescens Torr. \& Gray.

Oenothera triloba Nutt.

\section{Orobanche uniflora L.}

\section{Orobanchaceae}

\section{Oxalidaceae}

Oxalis corniculata L. Syn. = O. corniculata L. var. langloisii (Small) Wieg.

\#Oxalis dillenii Jacq.

${ }^{*}$ Oxalis stricta L. Syns. = O. europaea Jord. var. bushii (Small) Wieg. and O. europaea Jord. var. europaea. Wallis listed formas europaea, pilosella Wieg., and villicaulis Wieg.

Oxalis violacea $\mathrm{L}$. Syn. $=0$. violacea $\mathrm{L}$. var. tricnophora Fasaett. .

Papaveraceae

Argemone polyanthemos (Fedde) G.B.

Ownbey. Syn. $=$ A. intermedia auct. non

Sweet.

*\#Papaver dubium L.

Sanguinaria canadensis L. Syn. $=S$.

canadensis L. var. rotundifolia (Greene)

Fedde.

\section{Passifloraceae}

Passiflora incarnata L. Wallis listed formas alba Waterfall and incarnata.

Passiflora lutea L. Syn. = P. lutea L. var. glabriflora Fern.

\section{Phytolaccaceae}

Phytolacca americana L.

Rivina humilis $\mathrm{L}$. 
Plantago aristata Michx.

\section{Plantaginaceae}

\#Plantago elongata Pursh

\#Plantago heterophylla Nutt.

*Plantago lanceolata L.

\#Plantago major L.

\#Plantago patagonica Jacq.

Plantago pusilla Nutt.

\#Plantago rhodosperma Dcne.

Plantago rugelii Dcne.

Plantago virginica $L$.

\#Plantago wrightiana Dcne.

\section{Platanaceae}

Platanus occidentalis L.

\section{Podostemaceae}

\#Podostemum ceratophyllum Michx.

\#Phlox cuspidata Scheele

\section{Polemoniaceae}

Phlox divaricata L. ssp. laphamii (Wood)

Wherry. Syn. = P. divaricata L. var.

laphamii Wood.

Phlox pilosa L. ssp. ozarkana (Wherry) Wherry.

Syn. = P. pilosa L. var. ozarkana Wherry.

Phlox pilosa L. var. pilosa

Polemonium reptans $\mathrm{L}$.

\section{Polygalaceae}

Polygala ambigua Nutt. Syn. $=P$. verticillata $L$. var. dolichoptera Fern.

Polygala incarnata L.

Polygala sanguinea $\mathrm{L}$.

\#Polygala verticillata $\mathrm{L}$.

\section{Polygonaceae}

Eriogonum longifolium Nutt.

*\#Fagopyrum esculentum Moench

\#Polygonum amphibium L.

*Polygonum aviculare L. var. aviculare. Syn. = $P$. aviculare $L$. var. vegetum Ledeb.

Polygonum buxiforme Small. Syn. $=P$.

aviculare L. var. littorale (Link) W. D. J.

Koch.

*Polygonum convolvulus L.

*\#Polygonum cuspidatum Sieb. \& Zucc.
*Polygonum hydropiper L.

Polygonum hydropiperoides Michx. var.

hydropiperoides. Syns. $=P$.

hydropiperoides Michx. var. bushianum

Stanford and P. hydropiperoides Michx. var. opelousanum (Riddell ex Small)

Riddell ex W. Stone.

Polygonuum lapathifolium L.

*\#Polygonum orientale L.

Polygonum pensylvanicum L. var. pensylvanicum Syn. $=P$. pensylvanicum

L. var. laevigatum Fern.

Polygonum persicaria L.

Polygonum punctatum Ell. var. confertiflorum (Meisn.) Fassett

Polygonum punctatum Ell. var. leptostachyum ((Meisn.) Small

Polygonum punctatum Ell. var. punctatum

Polygonum ramosissimum Michx.

Polygonum sagittatum $\mathrm{L}$.

Polygonum scandens $\mathrm{L}$.

\#Polygonum setaceum Baldw.

Polygonum tenue Michx.

Polygonum virginianum $\mathrm{L}$. Syn. = Tovara virginiana (L.) Raf.

${ }^{*}$ Rumex acetosella $\mathrm{L}$.

Rumex altissimus Wood

${ }^{*}$ Rumex crispus $L$.

Rumex hastatulus Baldw.

${ }^{*}$ Rumex obtusifolius L.

*\#Rumex patientia L.

${ }^{*}$ Rumex pulcher $\mathrm{L}$.

\section{Portulacaceae}

Claytonia virginica $\mathrm{L}$.

Phemeranthus calycinus (Engelm.) Kiger. Syn. = Talinum calycinum Engelm.

Phemeranthus parviflorus (Nutt.) Kiger. Syn. = Talinum parviflorum Nutt.

Portulaca halimoides L. Syn. = Portulaca parvula Gray.

Portulaca oleracea L.

\#Portulaca pilosa L.

\section{Primulaceae}

*\#Anagallis arvensis L.

Androsace occidentalis Pursh 
Centunculus minimus $\mathrm{L}$.

Dodecatheon meadia L. ssp. meadia. Wallis listed formas album and meadia.

Lysimachia ciliata L.

Lysimachia lanceolata Walt.

Samolus valerandi L. ssp. parviflorus (Raf.) Hultén. Syn. = S. parviflorus Raf.

\section{Ranunculaceae}

Anemone caroliniana Walt. Wallis listed formas caroliniana and violacea Clute.

Anemone berlandieri Pritz. Syn. = Anemone decapetala auct. non Ard.

Anemone virginiana $\mathrm{L}$.

Aguilegia canadensis L. Syn. $=A$. canadensis

L. var. latiuscula (Greene) Munz.

Clematis ligusticifolia Nutt.

Clematis pitcheri Torr. \& Gray

*\#Clematis terniflora DC.

Clematis versicolor Small ex Rydb.

Clemiatis virginiana $\mathrm{L}$.

${ }^{*}$ Consolida ajacis (L.) Schur. Syn. =

Delphinium ajacis $\mathrm{L}$. Wallis listed formas ajacis and alba R. H. Cheney.

Delphinium carolinianum Walt. ssp. carolinianum. syn. $=D$. carolinianum Walt. var. crispum Perry and $D$. carolinianum Walt. var. nortonianum (Mack \& Bush) Perry.

Delphinium tricorne Michx. Wallis listed formas albiflora Millsp. and tricorne.

Enemion biternatum Raf. Syn. = Isopyrum biternatum (Raf.) Torr. \& Gray

Myosurus minimus $\mathrm{L}$.

Ranunculus abortivus L. var. abortivus

Ranunculus fascicularis Muhl. ex Bigelow Syn. $=R$. fascicularis Muhl. ex Bigelow var. aprica (Greene) Fern.

Ranunculus harveyi (Gray) Britt.

Ranunculus hispidus Michx.

Ranunculus hispidus Michx. var. nitidus

(Chapman) T. Duncan. Syn. $=R$. carolinianus DC.

Ranunculus laxicaulis (Torr.\& Gray) Darby

Ranunculus longirostris Godr.

\#Ranunculus macranthus Scheele

Ranunculus micranthus Nutt.
${ }^{*}$ Ranunculus parviflorus L.

Ranunculus pusillus Poir.

Ranunculus recurvatus Poir.

Ranunculus sceleratus $L$. var. sceleratus

Thalictrum dasycarpum Fisch. \& Avé-Lall. Syn. $=T$. dasycarpum Fisch. \& Avé-Lall. var. hypoglaucum (Rydb.) Boivin.

\#Thalictrum dioicum $\mathrm{L}$.

Thalictrum thalictroides (L.) Eames \& Boivin. Syn. = Anemonella thalictroides (L.) Eames \& Boivin.

\section{Rhamnaceae}

Berchemia scandens (Hill) K. Koch

Ceanothus americanus L. Syn. $=C$. americanus L. var. pitcheri Torr.\& Gray.

Ceanothus herbaceus Raf. Syn. $=C$. herbaceus Raf. var. pubescens (Torr. \& Gray ex S. Wats.) Shinners.

Frangula caroliniana (Walt.) Gray. Syn. = Rhamnus caroliniana Walt. var. caroliniana and Rhamnus caroliniana Walt. var. mollis Fern.

Rhamnus lanceolata Pursh ssp. glabrata (Gleason) Kartesz \& Gandhi. Syn. = Rhamnus lanceolata Pursh var. glabrata Gleason.

\section{Rosaceae}

Agrimonia parviflora Ait.

Agrimonia pubescens Wallr.

Agrimonia rostellata Wallr.

Amelanchier arborea (Michz. f.) Fern.

\#Amelanchier arborea (Michx. f.) Fern. var. alabamensis (Britt.) G.N. Jones

Aruncus dioicus (Walt.) Fern. var. pubescens (Rydb.) Fern.

Crataegus coccinioides Ashe

Crataegus crus-galli $\mathrm{L}$.

Crataegus engelmanni Sarg.

\#Crataegus intricata Lange

Crataegus mollis Scheele

\#Crataegus pruinosa (Wendl. f.) K. Koch

\#Crataegus punctata Jacq.

Crataegus reverchonii Sarg. Syn. $=C$. reverchoni Sarg. var. discolor (Sarg.) Palmer. 
Oklahoma Native Plant Record

Volume 7, Number 1, December 2007

Crataegus spathulata Michx.

Crataegus viridis $\mathrm{L}$.

*\#Duchesnea indica (Andr.) Focke

Fragaria virginiana Duchesne

Geum canadense Jacq. var. canadense. Syn.

$=\mathrm{G}$. canadense Jacq. var. camporum

(Rydb.) Fern. \& Weath.

Geum vernum (Raf.) Torr. \& Gray

Gillenia stipulata (Muhl. ex Willd.) Baill.

Malus ioensis (Wood) Britt. var. ioensis. Syn. = Pyrus ioensis (Wood) Bailey.

*Malus sylvestris P. Mill. Syn. = Pyrus malus $\mathrm{L}$.

Physocarpus opulifolius (L.) Maxim. var. intermedius (Rydb.) B.L. Robins.

Potentilla norvegica $\mathrm{L}$.

${ }^{*}$ Potentilla recta $\mathrm{L}$.

Potentilla simplex Michx. var. simplex

\#Prunus americana Marsh.

\#Prunus angustifolia Marsh.

\#Prunus gracilis Engelm. \& Gray

Prunus hortulana Bailey

Prunus mexicana S. Wats. Syn. $=P$. americana Marsh. var. lanata Sudsworth.

Prunus munsoniana W. Wright \& Hedrick.

${ }^{*}$ Prunus persica (L.) Batsch

Prunus rivularis Scheele. Syn. $=$ P. reverchonii

Sarg.

Prunus serotina Ehrh.

\#Prunus virginiana $\mathrm{L}$.

*\#Pyrus communis $L$.

Rosa carolina L. var. carolina. Syn. $=R$. carolina var. villosa (Best) Rehd. Wallis listed forma glandulosa (Crepin) Fern.

Rosa foliolosa Nutt. ex Torr. \& Gray

Rosa multiflora Thunb. ex Murr.

Rosa setigera Michx. var. setigera

Rosa setigera Michx. var. tomentosa Torr. \&

Gray

Rubus aboriginum Rydb.

\#Rubus argutus Link

Rubus allegheniensis Porter

Rubus bushii Bailey. Syn. = R. fructifer Bailey,

$R$. ozarkensis Bailey, and $R$. scibilis Bailey. \#Rubus flagellaris Willd.

Rubus frondosus Bigelow. Syn. = R. pratensis Bailey.
Rubus mollior Bailey

Rubus occidentalis L.

Rubus oklahomus Bailey

Rubus trivialis Michx.

Sanguisorba annua (Nutt. ex Hook.) Nutt. ex Torr. \& Gray

\section{Rubiaceae}

Cephalanthus occidentalis $\mathrm{L}$.

Diodia teres Walt. var. teres. Syn. $=D$. teres

Walt. var. setifera Fern. \& Grisc.

\#Diodia virginiana $\mathrm{L}$.

Galium aparine L. var. aparine. Syn. = G. aparine L. var. vaillantii (DC.) Koch.

Galium arkansanum Gray

Galium circaezans Michx. var. hypomalacum

Fern.

Galium concinnum Torr. \& Gray

\#Galium obtusum Bigelow

\#Galium pilosum Ait. var. pilosum

Galium pilosum Ait. var. puncticulosum

(Michx.) Torr. \& Gray

Galium tinctorium (L.) Scop.

Galium triflorum Michx. Wallis listed formas

glabrum Leyend and triflorum.

Galium virgatum Nutt.

Houstonia purpurea L. var. calycosa Gray.

Syn. = Houstonia lanceolata (Poir.) Britt.

Houstonia longifolia Gaertn.

Houstonia pusilla Schoepf. Syn. $=$ H. patens

Ell.

Houstonia purpurea L.

\#Houstonia rosea (Raf.) Terrell

\#Oldenlandia boscii (DC.) Chapman

*Sherardia arvensis $\mathrm{L}$.

Spermacoce glabra Michx.

Stenaria nigricans (Lam.) Terrell var. nigricans.

Syn. $=$ Houstonia nigricans (Lam.) Fern.

\section{Rutaceae}

Ptelea trifoliata $\mathrm{L}$.

\#Zanthoxylum americanum P. Mill.

\section{Salicaceae}

${ }^{*} \#$ Populus alba L.

Populus deltoides Marsh.

Salix caroliniana Michx.

Salix humilis Marsh. var. humilis. Syn. $=S$. 
humilis Marsh. var. hyporhysa Fern.

Salix interior Rowlee. Wallis listed forma

wheeleri (Rowlee) Rouleau.

Salix nigra Marsh.

\section{Santalaceae}

Comandra umbellata (L.) Nutt. ssp. umbellata.

Syn. $=$ C. richardsiana Fern .

\section{Sapindaceae}

\#Cardiospermum halicacabum L.

Sapindus saponaria L. var. drummondii (Hook. \& Arn.) L. Benson. Syn. = S. drummondii Hook. \& Arn.

\section{Sapotaceae}

Sideroxylon lanuginosum Michx. ssp. oblongifolium (Nutt.) T.D. Pennington. Syn. = Bumelia lanuginosa (Michx.) Pers. var. oblongifolia (Nutt.)

R. B. Clark.

\section{Saxifragaceae}

Heuchera americana L. var. hirsuticaulis (Wheelock) Rosendahl Butters \& Lakela

Penthorum sedoides L.

\#Saxifraga palmeri Bush

Saxifraga texana Buckl.

Saxifraga virginiensis Michx. var. subintegra

Goodman

\section{Saururus cernuus L.}

\section{Scrophulariaceae}

\#Agalinis densiflora (Benth.) Blake

Agalinis fasciculata (Ell.) Raf. Syn. = Gerardia

fasciculata Ell.

Agalinis gattingeri (Small) Small. Syn. =

Gerardia Gattingeri Small.

Agalinis heterophylla (Nutt.) Small ex Britt.

Syn. $=$ Gerardia heterophylla Nutt.

Agalinis tenuifolia (Vahl) Raf. var. parviflora

(Nutt.) Pennell. Syn. = Gerardia tenuifolia

Vahl. var. parviflora Nutt.

\#Agalinis viridis (Small) Pennell

Aureolaria grandiflora (Benth.) Pennell var. cinerea Pennell. Syn. = Gerardia

grandiflora Benth. var. cinerea (Pennell)

Cory.

\#Aureolaria pectinata (Nutt.) Pennell

Bacopa rotundifolia (Michx.) Wettst.

Buchnera americana L.

Castilleja coccinea (L.) Spreng. Wallis listed formas coccinea and lutescens Farwell.

\#Castilleja indivisa Engelm.

\#Castilleja purpurea (Nutt.) G. Don

\#Collinsia verna Nutt.

Collinsia violacea Nutt.

Dasistoma macrophylla (Nutt.) Raf. Syn. = Seymeria macrophylla Nutt.

Gratiola neglecta Torr.

Gratiola virginiana L.

*Kickxia elatine (L.) Dumort.

Leucospora multifida (Michx.) Nutt. Syn. = Conobea multifida (Michx.) Benth.

* Linaria vulgaris $\mathrm{P}$. Mill.

Lindernia dubia (L.) Pennell var. anagallidea (Michx.) Cooperrider. Syn. $=L$. anagallidea (Michx.) Pennell.

Lindernia dubia (L.) Pennell var. dubia

Mecardonia acuminata (Walt.) Small var. acuminata. Syn. = Bacopa acuminata (Walt.) B.L. Robins.

Mimulus alatus Ait.

Mimulus glabratus Kunth var. oklahomensis Fassett

\#Mimulus ringens $\mathrm{L}$.

Nuttallanthus texanus (Scheele) D.A. Sutton. Syn. = Linaria canadensis (L.) Dumont. var. texana (Scheele) Pennell.

Pedicularis canadensis L. ssp. canadensis.

Syn. $=P$. canadensis $L$. var. dobbsii Fern.

Penstemon arkansanus Pennell

Penstemon digitalis Nutt. ex Sims

\#Penstemon laxiflorus Pennell

Penstemon tubiflorus Nutt.

Scrophularia marilandica L. Wallis listed forma neglecta (Rydb.) Pennell.

*Verbascum blattaria L. Wallis listed formas albiflora (Don) House and blattaria.

*Verbascum thapsus L. \#Veronica anagallis-aquatica L. 
Oklahoma Native Plant Record

Volume 7, Number 1, December 2007

*Veronica arvensis L.

Veronica peregrina L. ssp. peregrine

Veronica peregrina L. ssp. xalapensis (Kunth)

Pennell. Syn. $=V$. peregrina L. var. xalapensis (Kunth) Pennell.

Veronicastrum virginicum (L.) Farw.

\section{Simaroubaceae}

*\#Ailanthus altissima (P. Mill.) Swingle

\section{Solanaceae}

${ }^{*}$ Datura inoxia P. Mill. Syn. = D. meteloides auct. p.p., non Dunal.

${ }^{*}$ Datura stramonium L.

Physalis angulata L. Syns. $=P$. angulata L . var. lanceifolia (Nees) Waterfall and $P$. angulata L. var. pendula (Rydberg) Waterfall.

Physalis cordata P. Mill. Syn. = P. pubescens L. var. glabra (Michx.) Waterfall.

\#Physalis hederifolia Gray

Physalis heterophylla Nees var. heterophylla

Physalis longifolia Nutt. var. longifolia. Syn. = Physalis virginiana Mill. var. sonorae (Torr.) Waterfall.

Physalis missouriensis Mackenzie \& Bush \#Physalis pubescens L. var. pubescens Physalis pubescens L. var. integrifolia (Dunal) Waterfall

Physalis pumila Nutt.

Physalis virginiana $P$. Mill. var. virginiana

Solanum americanum $P$. Mill.

Solanum carolinense $\mathrm{L}$. Wallis listed formas albiflorum (O. Ktze.) Benke and carolinense.

Solanum elaeagnifolium Cav.

Solanum rostratum Dunal

*Solanum physalifolium Rusby. Syn. = Solanum sarachoides auct. non Sendtner. \#Solanum ptychanthum Dunal

*\#Veronica persica Poir.

*\#Veronica polita Fries

Staphylea trifolia L.

\section{Tamaricaceae}

*\#Tamarix chinensis Lour.

*Tamarix gallica L.

\section{Tiliaceae}

Tilia americana L. var. americana. Syn. = Tilia neglecta Spach.

\#Tilia americana L. var. caroliniana (P. Mill.) Castigl.

\section{Ulmaceae}

Celtis laevigata Willd. var. laevigata

\#Celtis laevigata Willd. var. texana Sarg.

Celtis occidentalis L. Syn. = C. occidentalis L. var. pumila (Pursh) Gray.

Celtis tenuifolia Mitt. var. ternilfolia. Syn. = Celtis tenuifolia Nutt. var. georgiana. (Small) Fern. \& Schub.

Ulmus alata Michx.

Ulmus americana $\mathrm{L}$.

Ulmus crassifolia Nutt.

*Ulmus pumila L.

Ulmus rubra Muhl.

\#Ulmus serotina Sarg.

\section{Urticaceae}

Boehmeria cylindrica (L. ) Sw. var. cylindrica Laportea canadensis (L.) Weddell

Parietaria pensylvanica Muhl. ex Willd.

Pilea pumila (L.) Gray var. deamii (Lunell)

Fern.

Urtica chamaedryoides Pursh

Urtica dioica L.

\section{Valerianaceae}

Valerianella longiflora (Torr. \& Gray) Walp.

Valerianella ozarkana Dyal. Syn. $=$ V. bushii

Dyal.

Valerianella radiata (L.) Dufr.

\section{Callicarpa americana L}

Verbenaceae

Glandularia canadensis (L.) Nutt.

Glandularia pumila (Rydb.) Umber. Syn. = Verbena pumila Rydb.

Phryma leptostachya L. Formerly placed in the Phrymaceae. 
Phyla lanceolata (Michx.) Greene Syn. $=L$. lanceolata Michx. var. recognita Fern. \& Grisc.

Phyla nodiflora (L.) Greene. Syn. = L. incisa (Small) Tidestrom

Verbena bracteata Cav. ex Lag. \& Rodr.

Verbena halei Small

Verbena hastata L.

Verbena simplex Lehm.

Verbena stricta Vent. Wallis listed formas

albiflora Wadmond and stricta.

Verbena urticifolia L. var. urticifolia

\#Verbena urticifolia L. var. leiocarpa Perry \&

Fern.

\section{Violaceae}

Hybanthus concolor (T.E. Forst.) Spreng.

Viola bicolor Pursh. Syn. = V. kitaibeliana R. \&

S. var. rafinesquii (Greene) Fern.

Viola missouriensis Greene

\#Viola xpalmata L. (pro sp.) [brittoniana or pedatifida $\times$ affinis or sororia]

Viola pedata L. Syn. $=$ V. pedata L. var. lineariloba DC.

Viola pedatifida G. Don

Viola pubescens Ait. Syn. = V. pensylvanica

var. pensylvanica.

Viola pubescens Ait. var. scabriuscula

Schwein. ex Torr. \& Gray. Syn. $=V$.

pensylvanica Michx var. leiocarpa (Fern. \& Wieg.) Fern.

\#Viola X primulifolia

Viola sagittata Ait.

Viola sororia Willd. Syn. = V. papilionacea

Pursh.

Viola triloba Schwein. var. dilatata (EII.)

Brainerd

Viscaceae (=Loranthaceae)

Phoradendron leucarpum (Raf.) Reveal \& M.C. Johnston. Syn. $=P$. flavescens Nutt. ex Engelm.

Vitaceae

Ampelopsis arborea (L.) Koehne

Ampelopsis cordata Michx.

Cissus trifoliata (L.) L. Syn. = C. incisa (Nutt.)
Des Moulins.

Parthenocissus quinguefolia (L.) Planch. Wallis listed forma hirsuta (Donn) Fern.

\#Vitis aestivalis Michx. var. aestivalis

Vitis aestivalis Michx. var. bicolor Deam. Syn. $=V$. aestivalis Michx. var. argentifolia (Munson) Fern.

Vitis aestivalis Michx. var. lincecumii (Buckl.) Munson. Syn. $=V$. lincecumii Buckl. var. glauca Munson.

\#Vitis cinerea (Engelm.) Millard

\#Vitis palmata Vahl

\#Vitis riparia Michx.

\#Vitis rupestris Scheele

Vitis vulpina $\mathrm{L}$.

*Tribulus terrestris L.

\section{Monocotyledoneae \\ Acoraceae}

\#Acorus calamus L.

\section{Agavaceae}

Manfreda virginica (L.) Salisb. ex Rose. Syn. = Agave lata Shinners.

Yucca arkansana Trel.

Yucca filamentosa $\mathrm{L}$.

\#Yucca glauca Nutt.

Alisma subcordatum Raf.

Echinodorus cordifolius (L) Griseb.

Sagittaria ambigua J.G. Sm.

\#Sagittaria brevirostra Mackenzie \& Bush

Sagittaria calycina Engelm.

Sagittaria graminea Michx.

Sagittaria latifolia Willd. Wallis listed formas hastata (Pursh) Robins. and latifolia. Syn. $=$ S. latifolia Willd var. obtusa (Engelm.)

Wieg.

\#Sagittaria platyphylla (Engelm.) J.G. Sm.

\section{Araceae}

Arisaema triphyllum (L.) Schott ssp. triphyllum. Syn. = A. atrorubens (Ait.) Blume. Wallis formas virde (Engler) Fern. and zebrinum

Hoagland, B.W. 
Oklahoma Native Plant Record

Volume 7, Number 1, December 2007

(Sims) Fern.

Arisaema dracontium (L.) Schott

\section{Commelinaceae}

${ }^{*}$ Commelina communis $\mathrm{L}$.

Commelina diffusa Burm. $f$.

Commelina erecta L. var. angustifolia (Michx.)

Fern. Wallis listed forma crispa (Woot.)

Fern.

Commelina erecta L. var. deamiana Fern.

Commelina erecta L. var. erecta. Wallis listed formas erecta and intercursa Fern.

Commelina virginica $\mathrm{L}$.

Tradescantia ernestiana E.S. Anderson \& Woods. Wallis listed formas alba Waterfall and ernestiana.

\#Tradescantia hirsutiflora Bush

Tradescantia ohiensis Raf. Wallis listed forma ohiensis.

Tradescantia ozarkana E.S. Anderson \& Woods.

\section{Cyperaceae}

Bulbostylis capillaris (L.) Kunth ex C.B. Clarke ssp. capillaris. Syn. = Bulbostylis capillaris (L.) C. B. Clark var. crebra Fern.

Carex aggregata Mackenzie

\#Carex albicans Willd. ex Spreng. var. albicans

Carex amphibola Steud. var. amphibola

Carex annectens (Bickn.) Bickn.

Carex austrina Mackenzie

Carex bicknelii Britt.

Carex blanda Dewey

Carex brevior (Dewey) Mackenzie

Carex bulbostylis Mackenzie. Syn. = Carex amphibola Steud. var. globosa (Bailey)

Bailey.

Carex bushii Mackenzie

\#Carex caroliniana Schwein.

Carex cephalophora Muhl. var. cephalophora

Carex cherokeensis Schwein.

\#Carex complanata Torr. \& Hook.

Carex crawei Dewey

Carex crus-corvi Shuttlw. ex Kunze

\#Carex davisii Schwein. \& Torr.

\#Carex digitalis Willd.

\#Carex festucacea Schkuhr ex Willd.
\#Carex fissa Mackenzie

Carex flaccosperma Dewey

Carex frankii Kunth

Carex granularis Muhl. ex Willd. Syn. = Carex haleana Olney.

Carex gravida Bailey var. Iunelliana (Mackenzie) F.J. Herm.

Carex grayi Carey. Syn. $=$ Carex grayi Carey var. hispidula Gray.

Carex grisea Wahlenb. Syn. = Carex amphibola Steud var. turgida Fern.

Carex hirsutella Mackenzie

Carex hyalinolepis Steud.

Carex jamesii Schwein.

Carex leavenworthii Dewey. Syn. = Carex cephalophora Muhl. var. leavenworthii (Dewey) Kükenth.

Carex laevivaginata (Kükenth.) Mackenzie

\#Carex louisianica Bailey

Carex lupuliformis Sartwell ex Dewey

\#Carex lupulina Muhl. ex Willd.

Carex lurida Wahlenb.

\#Carex microdonta Torr. \& Hook.

\#Carex molestiformis Reznicek \& Rothrock

Carex muehlenbergii Schkuhr ex Willd. var. enervis Boott.

Carex muehlenbergii Schkuhr ex Willd. var. muehlenbergii.

Carex normalis Mackenzie

Carex oklahomensis Mackenzie. Syn. = Carex stipata Muhl. var. oklahomensis (Mackenzie) Gleason.

Carex oligocarpa Schkuhr ex Willd.

\#Carex oxylepis Torr. \& Hook.

Carex retroflexa Muhl. ex Willd.

\#Carex scoparia Schkuhr ex Willd.

\#Carex shinnersii P. Fothr. \& Reznicek

Carex shortiana Dewey

\#Carex socialis Mohlenbrock \& Schwegm.

\#Carex squarrosa L.

Carex triangularis Boeckl.

Carex vulpinoidea Michx.

Cyperus acuminatus Torr. \& Hook. ex Torr.

Cyperus bipartitus Torr. Syn. = Cyperus rivularis Kunth.

Cyperus echinatus (L.) Wood. Syns. = Cyperus ovularis (Michx.) Torr. var. 
ovularis and Cyperus ovularis (Michx.)

Torr. var. sphaericus Boeckl.

Cyperus erythrorhizos Muhl.

${ }^{*}$ Cyperus esculentus L.

Cyperus flavescens L. Syn. = Cyperus

flavescens L. var. poiformis (Pursh) Fern.

* \#yperus iria L.

Cyperus lupulinus (Spreng.) Marcks ssp.

lupulinus. Syn. = Cyperus filiculmis Vahl.

Cyperus odoratus $\mathrm{L}$.

\#Cyperus reflexus Vahl

\#Cyperus retroflexus Buckl. Syn. = Cyperus

uniflorus Torr. \& Hook.

\#Cyperus setigerus Torr. \& Hook.

Cyperus strigosus L. Syn. = Cyperus strigosus

L. var. robustior Britt.

Cyperus strigosus L. var. strigosus

Cyperus squarrosus L. Syn. = Cyperus

inflexus Muhl.

Cyperus virens Michx.

Eleocharis acicularis (L.) Roemer \& J.A.

Schultes var. acicularis Syn. = Eleocharis

acicularis (L.) Roemer \& J.A. Schultes var. gracilescens Svens.

Eleocharis engelmanni Steud. Wallis lists

forma englemanni.

\#Eleocharis erythropoda Steud.

Eleocharis lanceolata Fern.

Eleocharis macrostachya Britt.

Eleocharis montevidensis Kunth

Eleocharis obtusa (Willd.) J.A. Schultes

\#Eleocharis palustris (L.) Roemer \& J.A.

Schultes

\#Eleocharis parvula (Roemer \& J.A. Schultes)

Link ex Bluff, Nees \& Schauer

Eleocharis quadrangulata (Michx.) Roemer \&

J.A. Schultes

Eleocharis radicans (A. Dietr.) Kunth.

Eleocharis tenuis (Willd.) J.A. Schultes var. verrucosa (Svens.) Svens.

Fimbristylis annua (All.) Roemer \& J.A.

Schultes. Syn. = Fimbristylis baldwiniana (J.A. Schultes) Torr.

Fimbristylis autumnalis (L.) Roemer \& J.A. Schultes

Fimbristylis puberula (Michx.) Vahl var. puberula. Syn. = Fimbristylis drummondii
(Torr. \& Hook. ex Torr.) Boeckl.

Fimbristylis vahlii (Lam.) Link

Fuirena squarrosa Michx.

Isolepis carinata Hook. \& Arn. ex Torr. Syn. = Scirpus koilolepis (Steud.) Gleason.

Kyllinga pumila Michx. Syn. = Cyperus tenuifolius (Steud.) Dandy.

Lipocarpha drummondii (Nees) G. Tucker.

Syn. = Hemicarpha drummondii Nees.

Lipocarpha micrantha (Vahl) G. Tucker. Syn. =

Hemicarpha micrantha (Vahl) Pax.

Rhynchospora harveyi W. Boott

Rhynchospora macrostachya Torr. ex Gray

Rhynchospora recognita (Gale) Kral. Syn. =

Rhynchospora globularis (Chapm.) Small

var. recognita Gale.

\#Schoenoplectus acutus (Muhl. ex Bigelow)

A.\& D. Löve var. acutus

Schoenoplectus americanus (Pers.) Volk. ex

Schinz \& R. Keller. Syn. = Scirpus

americanus Pers. var. americanus.

Schoenoplectus californicus (C.A. Mey.) Palla.

Syn. = Scirpus californicus (C.A. Meyer)

Steud.

Schoenoplectus heterochaetus (Chase) Soják.

Syn. = Scirpus heterochaetus Chase.

Schoenoplectus tabernaemontani (K.C. Gmel.)

Palla. Syn. = Scirpus validus Vahl var.

creber Fern.

Scirpus atrovirens Willd.

Scirpus pendulus Muhl. Syn. = Scirpus

lineatus auct. non Michx.

Scleria ciliata Michx. var. ciliata

Scleria oligantha Michx.

Scleria pauciflora Muhl. ex Willd. var.

caroliniana (Willd.) Wood

Scleria triglomerata Michx.

\section{Dioscoreaceae}

${ }^{*}$ Dioscorea oppositifolia L. Syn. = Dioscorea batatas Dcne.

Dioscorea quaternata J.F. Gmel.

Dioscorea villosa $\mathrm{L}$. Wallis listed formas

glabrifolia (Bartlett) Fern. and villosa.

Hydrocharitaceae

Elodea nuttallii (Planch.) St. John

Hoagland, B.W. 


\section{Iridaceae}

*Belamcanda chinensis (L.) DC

Iris cristata Ait.

Iris virginica L. var. shrevei (Small) E. Anders.

Nemastylis nuttallii Pickering ex R.C. Foster

Sisyrinchium angustifolium P. Mill.

Sisyrinchium campestre Bickn. Wallis lists

forma kansanum (Bickn.)

Steyerm.

Sisyrinchium langloisii Greene. Syn. =

Sisyrinchium varians Bickn.

\section{Juncaceae}

Juncus acuminatus Michx.

Juncus biflorus Ell.

Juncus brachycarpus Engelm.

Juncus diffusissimus Buckl.

\#Juncus dudleyi Wieg.

Juncus effusus L. var. solutus Fern. \& Wieg.

Juncus interior Wieg.

Juncus marginatus Rostk.

Juncus nodatus Coville. Syn. = Juncus

acuminatus Michx. var. robustus Engelm.

Juncus scirpoides Lam.

\#Juncus secundus Beauv. ex Poir.

Juncus tenuis Willd.

Juncus torreyi Coville

Juncus validus Coville

Luzula bulbosa (Wood) Smyth \& Smyth

\#Luzula echinata (Small) F.J. Herm.

\section{Lemnaceae}

Lemna minor L.

Lemna valdiviana Phil.

Spirodela polyrrhiza (L.) Schleid.

\#Wolffia brasiliensis Weddell

\#Wolffia columbiana Karst.

Aletris farinosa $\mathrm{L}$.

Allium canadense $\mathrm{L}$. var. canadense

Allium canadense L. var. lavandulare (Bates)

Ownbey \& Aase

Allium canadense L. var. mobilense (Regel)

Ownbey

*\#Allium sativum L.
Allium stellatum Fraser ex Ker-Gawl.

${ }^{*}$ Allium vineale $\mathrm{L}$. Wallis listed formas compactum (Thuill.) Aschers and vineale.

${ }^{*}$ Asparagus officinalis $\mathrm{L}$.

Camassia scilloides (Raf.) Cory

Cooperia drummondii Herbert

Erythronium albidum Nutt.

Erythronium americanum Ker-Gawl.

Erythronium mesochoreum Knerr. Syn. =

Erythronium albidum Nutt. var. mesochoreum (Knerr) Rickett.

\#Erythronium rostratum W. Wolf

${ }^{*}$ Hemerocallis fulva (L.) L.

Hypoxis hirsuta (L.) Coville

Maianthemum racemosum (L.) Link ssp. racemosum. Syn. $=$ Smilacina racemosa

(L.) Desf. var. cylindrata Fern.

Nothoscordum bivalve (L.) Britt.

*\#Ornithogalum umbellatum L.

Polygonatum biflorum (Walt.) Ell. var. commutatum (J.A. \& J.H. Schultes)

Morong. Syn. = Polygonatum

canaliculatum auct. non (Muhl. ex Willd.)

Pursh.

Trillium recurvatum Beck

\#Trillium sessile L.

Trillium viridescens Nutt.

Uvularia grandiflora Sm.

Zigadenus nuttallii (Gray) S. Wats.

\section{Najadaceae}

Najas guadalupensis (Spreng.) Magnus

Orchidaceae

\#Calopogon oklahomensis D.H. Goldman

Calopogon tuberosus (L.) B.S.P. var. tuberosus. Syn. $=$ Calopogon pulchellus

R. Br. ex Ait. f.

\#Corallorrhiza odontorhiza (Willd.) Poir.

Corallorhiza wisteriana Conrad

\#Cypripedium kentuckiense C.F. Reed

\#Cypripedium parviflorum Salisb.

Hexalectris spicata (Walt.) Barnh.

\#Malaxis unifolia Michx.

Platanthera lacera (Michx.) G. Don. Syn. = Habenaria lacera (Michx.) R. Br.

Spiranthes cernua (L.) L.C. Rich. 
Spiranthes lacera (Raf.) Raf. var. gracilis

(Bigelow) Luer. Syn. = Spiranthes gracilis

(Bigelow) Beck.

Spiranthes tuberosa Raf. Syn. = Spiranthes

grayi Ames.

Spiranthes vernalis Engelm. \& Gray

\#Tipularia discolor (Pursh) Nutt.

Poaceae (= Gramineae)

*Aegilops cylindrica Host. Syn. = Aegilops

cylindrica Host. var. rubiginosa Popova.

${ }^{*}$ Agrostis gigantea Roth. Syn. = Agrostis alba

$\mathrm{L}$.

Agrostis elliottiana J.A. Schultes

Agrostis hyemalis (Walt.) B.S.P.

Agrostis perennans (Walt.) Tuckerman

\#Agrostis scabra Willd.

\#Agrostis stolonifera

*Aira caryophyllea L.

*\#Aira elegans Willd. ex Kunth

Alopecurus carolinianus Walt.

Andropogon gerardi Vitman. Syn. =

Andropogon gerardi Vitman var.

chrysocomas (Nash) Fern.

Andropogon ternarius Michx.

Andropogon virginicus L. var. virginicus. Syn.

= Andropogon virginicus L. var.

tetrastachyus (Ell.) Hack.

\#Aristida basiramea Engelm. ex Vasey

Aristida dichotoma Michx. var. curtissii Gray ex

S. Wats. \& Coult.

Aristida dichotoma Michx. var. dichotoma

Aristida longespica Poir. var. geniculata (Raf.)

Fern. Syn. = Aristida intermedia Scribn. \&

Ball.

Aristida longespica Poir. var. longespica

Aristida oligantha Michx.

Aristida purpurascens Poir.

*Arthraxon hispidus (Thunb.) Makino. Syn. =

Arthraxon hispidus (Thunb.) Makino var.

cryptatherus (Hack) Houda.

Arundinaria gigantea (Walt.) Muhl.

${ }^{*} \#$ Avena sativa $\mathrm{L}$.

Axonopus fissifolius (Raddi) Kuhlm. Syn. = Axonopus affinis Chase.

*Bothriochloa ischaemum (L.) Keng. Syn. = Andropogon ischaemum L.
Bothriochloa saccharoides (Sw.) Rydb. Syn. = Andropogon saccharoides Sw.

Bouteloua curtipendula (Michx.) Torr.

\#Brachyelytrum erectum (Schreb. ex Spreng.)

Beauv.

${ }^{*}$ Bromus catharticus Vahl.

*Bromus hordeaceus L. ssp. hordeaceus. Syn. $=$ Bromus mollis $\mathrm{L}$.

*Bromus japonicus Thunb.

Bromus pubescens Muhl. ex Willd.

${ }^{*}$ Bromus secalinus $\mathrm{L}$.

${ }^{*}$ Bromus tectorum L.

Cenchrus spinifex Cav. Syns. = Cenchrus incertus M.A. Curtis and Cenchrus pauciflorus Benth.

Chasmanthium latifolium (Michx.) Yates. Syn. = Uniola latifolia Michx.

Chloris verticillata Nutt.

\#Chloris virgata Sw.

Cinna arundinacea L. Syn. = Cinna

arundinacea L. var. inexpansa Fern. \&

Grisc.

Coelorachis cylindrica (Michx.) Nash. Syn. = Manisuris cylindrica (Michx.) Kuntze.

${ }^{*}$ Cynodon dactylon (L.) Pers.

${ }^{*}$ Dactylis glomerata $\mathrm{L}$.

Danthonia spicata (L.) Beauv. ex Roemer \&

J.A. Schultes. Syn. = Danthonia spicata

(L.) Beauv. ex Roemer \& J.A. Schultes var. longipila Scribn. \& Merr.

\#Diarrhena americana Beauv.

Diarrhena obovata (Gleason) Brandenburg.

Syn. = Diarrhena americana Beauv. var. obovata Gleason.

\#Dichanthelium aciculare (Desv. ex Poir.) Gould \& C. A. Clark

\#Dichanthelium acuminatum (Sw.) Gould \& C.A. Clark var. acuminatum

Dichanthelium acuminatum (Sw.) Gould \& C.A. Clark var. fasciculatum (Torr.) Freckmann.

Syn. = Panicum lanuginosum Ell. var. fasciculatum (Torr.) Fern.

\#Dichanthelium acuminatum (Sw.) Gould \&

C.A. Clark var. lindheimeri (Nash) Gould \&

C.A. Clark

Dichanthelium boscii (Poir.) Gould \& C.A. Clark. Syn. = Panicum boscii Poir. 
\#Dichanthelium clandestinum (L.) Gould

Dichanthelium commutatum (J.A. Schultes)

Gould. Syn. = Panicum commutatum

Schultes var. commutatum.

\#Dichanthelium depauperatum (Muhl.) Gould

\#Dichanthelium dichotomum (L.) Gould var. dichotomum

Dichanthelium latifolium (L.) Gould \& C.A. Clark. Syn. = Panicum latifolium L.

Dichanthelium laxiflorum (Lam.) Gould. Syn. = Panicum laxiflorum Lam.

Dichanthelium linearifolium (Scribn. ex Nash)

Gould. Syns = Panicum linearifolium

Scribn. var. linearifolium, Panicum

linearifolium Scribn. ex Nash var. werneri

(Scribn.) Fern., and Panicum perlongum

Nash.

Dichanthelium malacophyllum (Nash) Gould.

Syn. = Panicum malacophyllum Nash.

\#Dichanthelium oligosanthes (J.A. Schultes)

Gould var. oligosanthes

Dichanthelium oligosanthes (J.A. Schultes)

Gould var. scribnerianum (Nash) Gould.

Syns. = Panicum oligosanthes Schultes

var. helleri (Nash) Fern. and Panicum

oligosanthes Schultes var. scribnerianum

(Nash) Fern.

Dichanthelium ravenelii (Scribn. \& Merr.)

Gould. Syn. = Panicum ravenelii Scribn. \&

Merr.

Dichanthelium scoparium (Lam.) Gould. Syn.

= Panicum scoparium Lam.

Dichanthelium sphaerocarpon (Ell.) Gould.

Syn. = Panicum sphaerocarpon Ell.

Dichanthelium sphaerocarpon (Ell.) Gould var. isophyllum (Scribn.) Gould \& C.A. Clark.

Syn. = Panicum polyanthes Schultes.

Dichanthelium villosissimum (Nash)

Freckmann var. praecocius (A.S. Hitchc. \&

Chase) Freckmann. Syn. = Panicum

praecocius A.S. Hitchc. \& Chase.

Digitaria cognata (J.A. Schultes) Pilger. Syn. = Leptoloma cognatum (Schultes) Chase.

\#Digitaria ciliaris (Retz.) Koel.

Digitaria filiformis (L.) Koel.

*Digitaria ischaemum (Schreb.) Schreb. ex

Muhl. Syn. = Digitaria ischaemum
(Schreb.) Muhl.

Digitaria sanguinalis (L.) Scop.

\#Digitaria villosa (Walt.) Pers.

${ }^{*}$ Echinochloa colona (L.) Link. Wallis listed forma zonalis (Guss.) Wieg.

${ }^{*}$ Echinochloa crus-galli (L.) Beauv.

*Eleusine indica (L.) Gaertn.

Elymus canadensis $\mathrm{L}$.

Elymus hystrix L. Syn. = Hystrix patula Moench.

Elymus villosus Muhl. ex Willd.

Elymus virginicus L. var. virginicus. Syns. = Elymus virginicus L. var. glabriflorus (Vasey) Bush (Wallis listed formas australis (Scribn. \& Ball) Fern., hirsutiglumis (Scribn.) Fern., and virginicus) and Elymus virginicus L. var. jejunus (Ramaley) Rydb.

*\#Eragrostis barrelieri Daveau

Eragrostis capillaris (L.) Nees

${ }^{*}$ Eragrostis cilianensis (All.) Vign. ex Janchen.

Syn. = Eragrostis megastachya (Koel.)

Link.

Eragrostis curtipedicellata Buckl.

${ }^{*}$ Eragrostis curvula (Schrad.) Nees

Eragrostis frankii C.A. Mey. ex Steud.

Eragrostis hirsuta (Michx.) Nees

Eragrostis hypnoides (Lam.) B.S.P.

Eragrostis intermedia A.S. Hitchc.

Eragrostis pectinacea (Michx.) Nees ex Steud.

Eragrostis pilosa (L.) Beauv.

Eragrostis spectabilis (Pursh) Steud.

\#Eragrostis trichodes (Nutt.) Wood

Festuca paradoxa Desv.

Festuca subverticillata (Pers.) Alexeev. Syn. = Festuca obtusa Biehler.

\#Glyceria acutiflora Torr.

Glyceria striata (Lam.) A.S. Hitchc.

Gymnopogon ambiguus (Michx.) B.S.P.

*\#Holcus lanatus L.

Hordeum pusillum Nutt.

Koeleria macrantha (Ledeb.) J.A. Schultes.

Syn. = Koeleria cristata (L.) Pers.

Leersia oryzoides (L) Sw. Wallis listed formas glabra A.A. Eat. and oryzoides.

Leersia virginica Willd.

Leptochloa fusca (L.) Kunth ssp. fascicularis 
(Lam.) N. Snow. Syn. = Diplachne

fascicularis (Lam.) Beauv.

Leptochloa panicea (Retz.) Ohwi ssp. brachiata

(Steudl.) N. Snow. Syn. = Leptochloa

filiformis (Lam.) Beauv.

*Lolium perenne L. ssp. multiflorum (Lam.)

Husnot. Syn. = Lolium multiflorum Lam.

\#Melica mutica Walt.

Melica nitens (Scribn.) Nutt. ex Piper

*\#Microstegium vimineum (Trin.) A. Camus

\#Muhlenbergia bushii Pohl

Muhlenbergia capillaris (Lam.) Trin.

\#Muhlenbergia frondosa (Poir.) Fern.

Muhlenbergia schreberi J.F. Gmel.

Muhlenbergia sobolifera (Muhl. ex Willd.) Trin.

Muhlenbergia sylvatica Torr. ex Gray

\#Muhlenbergia tenuiflora (Willd.) B.S.P.

Nassella leucotricha (Trin. \& Rupr.) Pohl. Syn.

= Stipa leucotricha Trin. \& Rupr.

Neeragrostis reptans (Michx.) Nicora

Panicum anceps Michx.

Panicum brachyanthum Steud.

Panicum capillare L. var. capillare

Panicum dichotomiflorum Michx. var. dichotomiflorum

Panicum flexile (Gattinger) Scribn.

\#Panicum obtusum Kunth

\#Panicum philadelphicum Bernh. ex Trin.

Panicum rigidulum Bosc ex Nees var. rigidulum. Syn. $=$ Panicum agrostoides

Spreng. var. agrostoides.

Panicum virgatum $L$.

Pascopyrum smithii (Rydb.) A. Löve. Syn. = Agropyron smithii Rydb. var. smithii. \#Paspalidium geminatum (Forsk.) Stapf

*Paspalum dilatatum Poir.

Paspalum floridanum Michx. Syn. = Paspalum floridanum Michx var. glabratum Engelm.

Paspalum fluitans (Ell.) Kunth

Paspalum laeve Michx. Syn. = Paspalum laeve Michx var. pilosum Scribn.

Paspalum pubiflorum Rupr. ex Fourn. Syn. = Paspalum pubiflora Rupr. var. glabrum Vasey ex Scribn.

Paspalum setaceum Michx. Syn. $=$ Paspalum ciliatifolium Michx. var. ciliatifolium and Paspalum ciliatifolium Michx. var. muehlenbergii (Nash) Fern.

Phalaris caroliniana Walt.

*\#Phleum pretense $\mathrm{L}$.

${ }^{*}$ Poa annua $\mathrm{L}$.

\#Poa champaniana Scribn.

Poa pratensis $L$.

Poa sylvestris Gray

Polypogon interruptus Kunth

Schedonnardus paniculatus (Nutt.) Trel.

*\#Schedonorus phoenix (Scop.) Holub

*\#Schedonorus pratensis (Huds.) Beauv.

Schizachyrium scoparium (Michx.) Nash. Syn.

= Andropogon scoparius Michx.

\#Setaria leucopila (Scribn. \& Merr.) K. Schum.

Setaria parviflora (Poir.) Kerguélen. Syn. =

Setaria geniculata auct. non (Wild.) Beauv.

*Setaria pumila (Poir.) Roemer \& J.A. Schultes

ssp. pumila. Syn. = Setaria glauca (L.)

Beauv.

*Setaria viridis (L.) Beauv.

Sorghastrum nutans (L.) Nash

*Sorghum halepense (L.) Pers.

Spartina pectinata Bosc ex Link. Syn. =

Spartina pectinata Bosc ex Link var. suttiei

(Farw.) Fern.

Sphenopholis filiformis (Chapman) Scribn.

Sphenopholis intermedia (Rydb.) Rydb.

Sphenopholis obtusata (Michx.) Scribn. Syn. = Sphenopholis obtusata (Michx.) Scribn. var. lobata (Trin.) scribn. Wallis listed forma lobata.

Sporobolus clandestinus (Biehler) A.S. Hitchc. Syn. = Sporobolus canovirens Nash.

Sporobolus compositus (Poir.) Merr. var. compositus. Syn. $=$ Sporobolus asper (Beauv.) Kunth var. hookeri (Trin.) Vasey.

\#Sporobolus compositus (Poir.) Merr. var. drummondii (Trin.) Kartesz \& Gandhi

Sporobolus cryptandrus (Torr.) Gray

Sporobolus heterolepis (Gray) Gray

Sporobolus vaginiflorus (Torr. ex Gray) Wood var. vaginiflorus

\#Sporobolus vaginiflorus (Torr. ex Gray) Wood var. ozarkanus (Fern.) Shinners

Steinchisma hians (Ell.) Nash. Syn. = Panicum hians Ell.

Tridens muticus (Torr.) Nash var. elongatus

Hoagland, B.W. 
(Buckl.) Shinners. Syn. = Triodia elongata (Buckl.) Scribn.

Tridens flavus (L.) A.S. Hitchc. Syn. = Triodia flava (L.) Smyth.

Tridens strictus (Nutt.) Nash. Syn. = Triodia stricta (Nutt.) Benth. ex Vasey.

Triplasis purpurea (Walt.) Chapman

Tripsacum dactyloides (L.) L.

${ }^{*}$ Triticum aestivum $\mathrm{L}$.

\#Urochloa platyphylla (Munro ex Wright) R. Webster

*Vulpia myuros (L.) K.C. Gmel.

Vulpia octoflora (Walt.) Rydb.

\#Zizaniopsis miliacea (Michx.) Doell \& Aschers.

\section{Pontederiaceae}

\#Heteranthera dubia (Jacq.) MacM.

Heteranthera limosa (Sw.) Wild.

Potamogetonaceae (= Zosteraceae)

*Potamogeton crispus L.

Potamogeton diversifolius Raf.

Potamogeton foliosus Raf. ssp. foliosus

Potamogeton nodosus Poir.

\section{Smilacaceae}

Smilax bona-nox L. Syn. = Smilax bona-nox L. var. hastata (Willd.) A. DC. and Smilax bona-nox L. var. hederifolia (Bey.) Fern. \#Smilax ecirrata (Engelm. ex Kunth) S. Wats. Smilax glauca Walt. Syn. = Smilax glauca Walt. var. leucophylla Blake.

\#Smilax herbacea L.

Smilax lasioneura Hook.

Smilax pulverulenta Michx.

\#Smilax rotundifolia L.

Smilax tamnoides L. Syn. = Smilax tamnoides L. var. hispida (Muhl. ex Torr.) Fern.

\section{Sparganiaceae}

\#Sparganium americanum Nutt. Sparganium androcladum (Engelm.) Morong

*Typha angustifolia L.

\section{Typhaceae}

Typha domingensis Pers.

Typha latifolia L. Wallis listed formas ambigua (Sonder) Kronf. and latifolia.

\section{Zannichelliaceae}

Zannichellia palustris $\mathrm{L}$. Formerly a member of the Zosteraceae. 